\title{
The Robbin-Salamon index theorem in Banach spaces with UMD
}

\author{
Patrick J. Rabier \\ Communicated by Charles Li, received June 23, 2004.
}

\begin{abstract}
Let $(A(t))_{t \in \mathbf{R}}$ be a continuous family of unbounded operators on a UMD Banach space $X$, with $t$-independent domain $W$ dense and compactly embedded in $X$. Under these and other general technical conditions, we prove that the operator $D_{A}=\frac{d}{d t}-A(\cdot)$ is a Fredholm operator between the spaces $W^{1, p}(\mathbf{R}, X) \cap L^{p}(\mathbf{R}, W)$ and $L^{p}(\mathbf{R}, X)$ for every $p \in(1, \infty)$. We also characterize the index of $D_{A}$ by the spectral flow of $A$. These results generalize those obtained by Robbin and Salamon when $X$ and $W$ are Hilbert spaces, $p=2$ and the family $(A(t))_{t \in \mathbf{R}}$ is selfadjoint. The hypotheses involved are satisfied by broad classes of second order elliptic differential operators with boundary conditions and by PDE systems with a Hamiltonian-like structure. An application to the $L^{p}$ maximal regularity for the nonautonomous Cauchy problem on the half-line is discussed in detail.
\end{abstract}

\section{Contents}

1. Introduction 304

2. Background material 307

3. The semi-Fredholm property 311

4. Regularity and the Fredholm property 314

5. Elementary properties of the index of $D_{A} \quad 317$

6. Calculation of the index of $D_{A} \quad 319$

7. The case of differential operators 328

8. Initial value problems on the half-line and $L^{p}$ maximal regularity 330

References 336

1991 Mathematics Subject Classification. Primary 46; Secondary 47, 35.

Key words and phrases. Fredholm and semi-Fredholm operators, unbounded operator, Banach space with UMD, Rademacher boundedness, holomorphic semigroup, elliptic differentia operator, Hamiltonian system. 


\section{Introduction}

If $H$ is a Hilbert space and $(A(t))_{t \in \mathbf{R}}$ is a $C^{1}$ family of unbounded selfadjoint operators on $H$ with invertible limits $A_{ \pm}$as $t \rightarrow \pm \infty$ and a $t$-independent dense domain $D(A(t))=W$ compactly embedded in $H$, Robbin and Salamon [34] have shown that the operator $D_{A}$ defined by $D_{A} u:=\frac{d u}{d t}-A u$ is Fredholm from $W^{1,2}(\mathbf{R}, H) \cap L^{2}(\mathbf{R}, W)$ to $L^{2}(\mathbf{R}, H)$. The index of $D_{A}$ generalizes the difference between the Morse indices of $A_{+}$and $A_{-}$even though these indices themselves do not exist if both the positive and negative spectra of $A_{ \pm}$are infinite. A typical example (Atiyah, Patodi and Singer [8]), with numerous variants, arises when $(A(t))_{t \in \mathbf{R}}$ is a family of (selfadjoint) elliptic operators on a compact manifold.

In this paper, the Fredholm property of $D_{A}$ is established for continuous families of non-selfadjoint unbounded operators in suitable Banach spaces and in the " $L$ " setting. Partial results of this type (with $D(A(t))$ possibly $t$-dependent) have recently been obtained by Di Giorgio, Lunardi and Schnaubelt [16], via exponential dichotomies. In this spirit, see also Latushin and Tomilov [26] and Di Giorgio and Lunardi $[\mathbf{1 5}]$ for the case of Hölder rather than Sobolev spaces. The recent work by Abbondandolo and Majer [1] addresses the case when $X$ is a Hilbert space and $A(t)$ is bounded. When $\operatorname{dim} X=\infty$, this is a much different problem than the one considered here.

Some preliminary discussion is needed before the appropriate hypotheses can be formulated. An important generalization of Hilbert space is found in the concept of Banach space with UMD (unconditionality of martingale differences), introduced by Burkholder [11] in 1966. While their original definition was motivated by probability theory, such spaces happen to provide an adequate framework for the discussion of evolution problems. Closed subspaces, products and duals of Banach spaces with UMD are themselves Banach spaces with UMD. Also, Banach spaces with UMD are (super)reflexive (Maurey [28], Aldous [5]). Hilbert spaces as well as the $L^{q}$ spaces, $q \in(1, \infty)$, have the UMD property $([\mathbf{1 1}],[\mathbf{1 2}$, Section 3$])$.

The characterization of the UMD property in terms of the Hilbert transform (Burkholder [13], Bourgain [9]) led Bourgain [10] and later Zimmermann [40] to obtain operator-valued versions of various $L^{p}$ multiplier theorems, notably Mikhlin's, in Banach spaces with UMD, but only for scalar multiples of the identity. This limitation was recently removed by Weis $[\mathbf{3 8}]$, which enabled the author to prove a generalization of the Robbin-Salamon theorem in the UMD setting when the family $A(t)$ is constant $([\mathbf{3 2}])$. This generalization is one of the main tools needed here to discuss the general case.

A key ingredient in Weis' multiplier theorem is the combination of the UMD property of the underlying Banach space with an emerging concept of boundedness for families of operators. This so-called "Rademacher boundedness" ( $\mathrm{r}$ boundedness for short) is captured by the following definition, in which $r_{k}(t):=$ $\operatorname{sgn} \sin 2^{k-1} \pi t, k \in \mathbf{N}$, denotes the sequence of Rademacher functions on $[0,1]$.

Definition 1.1. Let $X$ and $Y$ be Banach spaces. The subset $\mathcal{T} \subset \mathcal{L}(X, Y)$ is said to be Rademacher-bounded (r-bounded) if there is a constant $C \geq 0$ such that

$$
\int_{0}^{1}\left\|\sum_{k=1}^{\kappa} r_{k}(\tau) T_{k} x_{k}\right\|_{Y} d \tau \leq C \int_{0}^{1}\left\|\sum_{k=1}^{\kappa} r_{k}(\tau) x_{k}\right\|_{X} d \tau
$$


for every finite collections $T_{1}, \ldots, T_{\kappa} \in \mathcal{T}$ and $x_{1}, \ldots, x_{\kappa} \in X$. The smallest constant $C$ for which (1.1) holds is called the r-bound of $\mathcal{T}$, denoted by $r(\mathcal{T})$ or by $r_{\mathcal{L}(X, Y)}(\mathcal{T})$ if it is important to specify that $\mathcal{T}$ is viewed as a subset of $\mathcal{L}(X, Y)$.

By letting $\kappa=1$ in Definition 1.1, every r-bounded subset $\mathcal{T} \subset \mathcal{L}(X, Y)$ is bounded and $\sup _{T \in \mathcal{T}}\|T\| \leq r(\mathcal{T})$. For $p, q \in[1, \infty)$, the Khintchin-Kahane inequality (Lindenstrauss and Tzafriri [27, Part II, p. 74]) ensures the existence of a constant $A_{p, q}>0$ such that

$$
\left(\int_{0}^{1}\left\|\sum_{k=1}^{\kappa} r_{k}(\tau) x_{k}\right\|_{X}^{p} d \tau\right)^{\frac{1}{p}} \leq A_{p, q}\left(\int_{0}^{1}\left\|\sum_{k=1}^{\kappa} r_{k}(\tau) x_{k}\right\|_{X}^{q} d \tau\right)^{\frac{1}{q}} .
$$

in any Banach space $X$. As a result, Definition 1.1 is unaffected if $\int_{0}^{1}\|\cdot\| d \tau$ is replaced by $\left(\int_{0}^{1}\|\cdot\|^{p} d \tau\right)^{\frac{1}{p}}$ in $(1.1)$, for any $p \in[1, \infty)$. In particular, with $p=2$, the orthonormality of the Rademacher functions shows that r-boundedness is the same as boundedness if $X$ and $Y$ are Hilbert spaces. In general Banach spaces (even with UMD), r-boundedness is more restrictive than ordinary boundedness.

Remark 1.1. If $X=Y$ is an $L^{q}$ space, $q \in[1, \infty)$, there is a more convenient equivalent definition of r-boundedness which does not involve the Rademacher functions: $\mathcal{T} \subset \mathcal{L}\left(L^{q}\right)$ is r-bounded if and only if there is a constant $C>0$ such that

$$
\left\|\left(\sum_{k=1}^{\kappa}\left|T_{k} f_{k}\right|^{2}\right)^{\frac{1}{2}}\right\|_{L^{q}} \leq C\left\|\left(\sum_{k=1}^{\kappa}\left|f_{k}\right|^{2}\right)^{\frac{1}{2}}\right\|_{L^{q}},
$$

for every finite collections $T_{1}, \ldots, T_{\kappa} \in \mathcal{T}$ and $f_{1}, \ldots, f_{\kappa} \in L^{q}$. This follows at once from [27, Part II, Theorem 1.d.6, p. 49]. Evidently, the only difference with normboundedness is that $C$ must be independent of $\kappa$. More generally, a similar formulation is valid in q-convex Banach lattices.

If $A_{0}$ is an unbounded operator on a Banach space $X$, then $\sigma\left(A_{0}\right)$ and $R\left(A_{0}, \lambda\right)$ will denote the spectrum and resolvent of $A_{0}$, respectively. Because of spectral considerations, it is convenient to work with complex spaces, but the main theorems remain true in the real case as well (Remark 6.2).

After these preliminaries, we are now in a position to state our generalization of the Robbin-Salamon theorem. From now on, $X$ is a Banach space with UMD and $W \subset X$ is a normed space. Given a family $(A(t))_{t \in \mathbf{R}}$ of unbounded operators on $X$ with common domain $W$, we shall assume that the following conditions hold:

(H1) $W$ is a Banach space and the embedding $W \hookrightarrow X$ is compact and dense.

(H2) $A \in C^{0}(\mathbf{R}, \mathcal{L}(W, X))$.

(H3) There are operators $A_{+}, A_{-} \in G L(W, X)$ such that

$$
\lim _{t \rightarrow \infty}\left\|A(t)-A_{+}\right\|_{\mathcal{L}(W, X)}=\lim _{t \rightarrow-\infty}\left\|A(t)-A_{-}\right\|_{\mathcal{L}(W, X)}=0 .
$$

The hypothesis (H3) justifies the notation $A_{-}=A(-\infty), A_{+}=A(\infty)$, convenient to formulate further assumptions. As usual, $\overline{\mathbf{R}}:=[-\infty, \infty]$. The operators $A_{ \pm}$are also viewed as unbounded operators on $X$ with domain $W$. The next condition controls the behavior of the resolvent of $A(t)$ on the imaginary axis. It is vacuous in the selfadjoint case (see below). 
(H4) For every $t \in \overline{\mathbf{R}}$ there is a constant $C_{0}(t)>0$ such that, for some $n_{0}(t) \in$ $\mathbf{N}$,

$$
r_{\mathcal{L}(X)}\left(\left\{2^{n} a R\left(i 2^{n} a, A(t)\right): n \geq n_{0}(t)\right\}\right) \leq C_{0}(t), \quad a \in \mathbf{R}, 1 \leq|a| \leq 2,
$$

where the left-hand side of (1.3) refers to the r-bound of the set $\left\{2^{n} a R\left(i 2^{n} a, A(t)\right)\right.$ : $\left.n \geq n_{0}(t)\right\} \subset \mathcal{L}(X)$ (see Definition 1.1). This implies

$$
\|\xi R(i \xi, A(t))\|_{\mathcal{L}(X)} \leq C_{0}(t), \quad \xi \in \mathbf{R},|\xi| \geq r_{0}(t),
$$

with $r_{0}(t)=2^{n_{0}(t)}$. When $X$ is a Hilbert space, (1.3) is equivalent to (1.4) holding for some $C_{0}(t)>0$ and $r_{0}(t)>0$. For clarity, it should be pointed out that (1.3) holds if $r_{\mathcal{L}(X)}\left(\left\{\xi R(i \xi, A(t)):|\xi| \geq 2^{n_{0}(t)}\right\}\right) \leq C_{0}(t)$, but the latter assumption is stronger (in general, the r-bound of a union is larger than the maximum of the r-bounds)

In our framework, the invertibility of the limits $A_{ \pm}$does not suffice. The appropriate substitute is the stronger condition that

(H5) $\sigma\left(A_{ \pm}\right) \cap i \mathbf{R}=\emptyset$.

The hypotheses (H1) to (H3), plus some differentiability of $A$, are retained in [34] when $X$ and $W$ are Hilbert spaces and $A(t)$ is selfadjoint. If so, (H4) and (H5) also hold: (1.3) has the form (1.4), which in turn is well known to be true for selfadjoint operators. Thus, (H4) holds and (H5) follows from the invertibility of $A_{-}$and $A_{+}$in (H3).

Remark 1.2. The case when $W=X$ and hence $(A(t))_{t \in \mathbf{R}}$ is bounded on $X$ is also instructive: (H1) amounts to $\operatorname{dim} X<\infty$ and the other assumptions reduce to (H2), (H3) and (H5). On the other hand, it is not difficult to find bounded counterexamples to Theorem 1.1 below when $\operatorname{dim} X=\infty$ and the compactness of the embedding of $W=X$ into $X$ is the only hypothesis that breaks down. See for instance [1].

Except for (H4), the assumptions made above can easily be checked directly in many concrete examples, notably from elliptic PDEs. We shall postpone comments about the practical verification of (H4) until Section 7.

The main result of this paper may be summarized by

THEOREM 1.1. If the family $(A(t))_{t \in \mathbf{R}}$ of unbounded operators on $X$ satisfies the assumptions (H1) to (H5), then the operator $D_{A}:=\frac{d}{d t}-A$ is Fredholm from $W^{1, p}(\mathbf{R}, X) \cap L^{p}(\mathbf{R}, W)$ to $L^{p}(\mathbf{R}, X)$ for every $p \in(1, \infty)$.

The proof of Theorem 1.1 proceeds in two main steps: In Section 3, we show that the operator $D_{A}$ is semi-Fredholm of index $\nu \in \mathbf{Z} \cup\{-\infty\}$. The proof relies upon the isomorphism theorem of [32] via suitable estimates. While similar estimates were already used in [34], they are here obtained without selfadjointness or differentiability assumption and by a quite different line of arguments.

The second step (Section 4) consists in proving that the first step is valid for $D_{-A^{*}}$ and that ker $D_{-A^{*}}$ is the annihilator of the range of $D_{A}$. This is mostly an issue of regularity of the weak solutions for $D_{-A^{*}}$, but it is simpler than the analogous step in [34] in spite of the fact that our framework is much more general. The difference lies in the choice for the adjoint operator: In this paper, $A^{*}(t)$ is the adjoint of $A(t) \in \mathcal{L}(W, X)$, so that $A^{*}(t) \in \mathcal{L}\left(X^{*}, W^{*}\right)$ is viewed as an unbounded operator on $W^{*}$ with domain $X^{*}$. In contrast, the Robbin-Salamon 
approach leads to viewing $A^{*}(t)$ as an unbounded operator on $X^{*}$ with smaller domain $D\left(A^{*}(t)\right):=\left\{x^{*} \in X^{*}: A^{*}(t) x^{*} \in W^{*}\right.$ is continuous for the topology of $X\} \subset X^{*}$. This difference remains important when $X$ is a Hilbert space and the UMD property and Rademacher boundedness considerations are not an issue, because working with a smaller domain requires proving stronger regularity results. In particular, in the Hilbert and selfadjoint case, our proof is simpler than that of Robbin and Salamon, yet valid for merely continuous families $(A(t))_{t \in \mathbf{R}}$ (a question explicitly left open in [34]) and for $p \in(1, \infty)$ rather than just $p=2$.

Relatively elementary properties of the index of $D_{A}$ are established in Section 5 , notably its $p$-independence (Theorem 5.1), sufficient conditions for $D_{A}$ to have index 0 (Theorem 5.2 and Corollary 5.4) and a theorem of invariance of the index under perturbations which may be neither compact nor limited in size (Theorem 5.3 and Remark 5.1).

In Section 6, we show that the index of $D_{A}$ can be viewed as the algebraic count of the eigenvalues of $A(t)$ crossing the imaginary axis as $t$ runs from $-\infty$ to $\infty$ (spectral flow). This generalizes the characterization given in [34] and is very useful to calculate the index of $D_{A}$ in nontrivial examples. In Section 7 , we discuss how the hypotheses (H1) to (H5) can be checked when $(A(t))_{t \in \mathbf{R}}$ is a family of second order elliptic operators on a bounded domain and also stress the relevance of Theorem 1.1 for systems of PDEs with a Hamiltonian-like structure.

The last section shows how the Fredholm properties of $D_{A}$ on the whole line can be used to obtain similar properties for initial value problems on the half-line $\mathbf{R}_{+}$and how this has a direct impact on the famous $L^{p}$ maximal regularity question for the nonautonomous Cauchy problem.

In the next section, we develop some material needed in various places to carry out the program outlined above. Most results there are either collected or derived from $[\mathbf{3 2}]$.

\section{Background material}

We begin with a list of useful consequences of the hypotheses (H1) to (H5).

THEOREM 2.1. Under the assumptions (H1) to (H4), the following properties hold for every $t \in \overline{\mathbf{R}}$ :

(i) If $\lambda \in \mathbf{C} \backslash \sigma(A(t))$, then, $A(t)-\lambda I \in G L(W, X)$.

(ii) $A(t)$ has compact resolvent (hence $\sigma(A(t))$ is discrete).

(iii) The norm of $W$ is equivalent to the graph norm of $A(t)$ (hence $A(t)$ is a closed operator on $X$ since $W$ is a Banach space).

In addition,

(iv) If $X$ is has the UMD property, then $W$ has the UMD property.

Proof. (i) By the continuity of the embedding $W \hookrightarrow X$ in (H1), $A(t)-\lambda I \in$ $\mathcal{L}(W, X)$ and $A(t)-\lambda I$ is a bijection of $W$ onto $X$ since $\lambda \notin \sigma(A(t))$. Thus, $A(t)-\lambda I \in G L(W, X)$ by the inverse mapping theorem.

(ii) From (i), $(A(t)-\lambda I)^{-1} \in \mathcal{L}(X, W)$. Since, by (H1), the embedding $W \hookrightarrow X$ is compact, it follows that $(A(t)-\lambda I)^{-1} \in \mathcal{L}(X)$ is compact. Thus, $A(t)$ has compact resolvent and $\sigma(A(t))$ is discrete since $\sigma(A(t)) \neq \mathbf{C}$ by (1.4) (implied by (H4)).

\footnotetext{
${ }^{1}$ It is not difficult to see that, due to (H3), this equivalence is in fact uniform in $t \in \overline{\mathbf{R}}$.
} 
(iii) Given $t \in \overline{\mathbf{R}}$, the graph norm of $A(t)$ is equivalent to the graph norm of $A(t)-\lambda I$ for every $\lambda \in \mathbf{C}$. It thus follows from (i) and (ii) that we may assume that $A(t) \in G L(W, X)$ with no loss of generality. If so, there is a constant $C=C(t)>0$ such that $C^{-1}\|w\|_{W} \leq\|A(t) w\|_{X} \leq C\|w\|_{W}$ for every $w \in W$. This implies $C^{-1}\|w\|_{W} \leq\|A(t) w\|_{X}+\|w\|_{X} \leq C\|w\|_{W}+\|w\|_{X}$. After increasing $C$ if necessary, we may assume that $\|w\|_{X} \leq C\|w\|_{W}$ since the embedding $W \hookrightarrow X$ is continuous by (H1). Thus, $C^{-1}\|w\|_{W} \leq\|A(t) w\|_{X}+\|w\|_{X} \leq 2 C\|w\|_{W}$ for $w \in W$.

(iv) If $X$ has the UMD property, the same thing is true of $X \times X$ and of every closed subspace of $X \times X$ (see the Introduction). By (iii), $W$ is isomorphic to a closed subspace of $X \times X$ (the graph of $A(t)$ for any chosen $t$ ) and hence has the UMD property.

The following result is a sharpening of Theorem 1.1 when $A(t)$ is constant.

TheOrem 2.2. ([32]) Let $X$ be a Banach space with UMD and let $A_{0}$ be a closed unbounded operator on $X$ with domain $D\left(A_{0}\right)=W$ dense in $X$ and equipped with the graph norm of $A_{0}$. Suppose that:

(i) $\sigma\left(A_{0}\right) \cap i \mathbf{R}=\emptyset$.

(ii) There is a constant $C_{0}>0$ such that, for some $n_{0} \in \mathbf{N}$,

$$
r_{\mathcal{L}(X)}\left(\left\{2^{n} a R\left(i 2^{n} a, A_{0}\right): n \geq n_{0}\right\}\right) \leq C_{0}, \quad \forall a \in \mathbf{R}, 1 \leq|a| \leq 2 .
$$

Then, the operator $\frac{d}{d t}-A_{0}$ is an isomorphism of $W^{1, p}(\mathbf{R}, X) \cap L^{p}(\mathbf{R}, W)$ onto $L^{p}(\mathbf{R}, X)$ for every $p \in(1, \infty)$.

REMARK 2.1. More generally, $\frac{d}{d t}-A_{0}$ is an isomorphism of $W^{k+1, p}(\mathbf{R}, X) \cap$ $W^{k, p}(\mathbf{R}, W)$ onto $W^{k, p}(\mathbf{R}, X)$ for every $p \in(1, \infty)$ and every $k \in \mathbf{N} \cup\{0\} ;$ see $[\mathbf{3 2}$, Remark 4.3].

Unlike Theorem 1.1, Theorem 2.2 is valid even if the embedding $W=D\left(A_{0}\right) \hookrightarrow$ $X$ is not compact. Recall (see e.g. Kato [25]) that if $X$ is a Banach space and $A_{0}, B$ are unbounded linear operators on $X$, then $B$ is said to be $A_{0}$-bounded if $D\left(A_{0}\right) \subset D(B)$ and there are constants $\alpha, \beta \geq 0$ such that

$$
\|B x\|_{X} \leq \alpha\left\|A_{0} x\right\|_{X}+\beta\|x\|_{X}, \quad x \in D\left(A_{0}\right) .
$$

The infimum of the possible constants $\alpha$ above is called the $A_{0}$-bound $\alpha(B)$ of $B$ or relative bound of $B$. It is rather fortunate that the technical condition (ii) in Theorem 2.2 is unaffected by relatively bounded perturbations with small enough relative bounds:

TheOrem 2.3. ([32]) Let $X$ be a Banach space and let $A_{0}$ be a closed unbounded operator on $X$. Suppose that there is a constant $C_{0}>0$ such that, for some $n_{0} \in \mathbf{N}$,

$$
r_{\mathcal{L}(X)}\left(\left\{2^{n} a R\left(i 2^{n} a, A_{0}\right): n \geq n_{0}\right\}\right) \leq C_{0}, \quad \forall a \in \mathbf{R}, 1 \leq|a| \leq 2 .
$$

Then, there is $\alpha_{0}>0$ such that (2.3) continues to hold with $A_{0}$ replaced by $A_{0}+H$ whenever $H$ is an $A_{0}$-bounded operator on $X$ with $A_{0}$-bound $\alpha(H)<\alpha_{0}$. More precisely, given $(\alpha, \beta)$ such that $\alpha \in\left(0, \alpha_{0}\right)$ and $\beta \geq 0$, there are $C(\alpha)>0$ and $n(\alpha, \beta) \in \mathbf{N}$, both independent of $H$ satisfying (2.2), such that ${ }^{2}$

$$
r_{\mathcal{L}(X)}\left(\left\{2^{n} a R\left(i 2^{n} a, A_{0}+H\right): n \geq n(\alpha, \beta)\right\}\right) \leq C(\alpha), \quad \forall a \in \mathbf{R}, 1 \leq|a| \leq 2 .
$$

\footnotetext{
${ }^{2}$ Of course, $C(\alpha)$ and $n(\alpha, \beta)$ also depend upon $A_{0}$.
} 
The following corollary shows that the assumptions (H1) through (H5) are unaffected by small enough perturbations of $A(t)$. It will be useful in Section 6 .

Corollary 2.4. Suppose that (H1) to (H5) hold. There is $\varepsilon>0$ such that if $H \in C^{0}(\mathbf{R}, \mathcal{L}(W, X))$ satisfies $\|H(t)\|_{\mathcal{L}(W, X)}<\varepsilon$ and $\lim _{t \rightarrow \pm \infty} H(t)=0$, then (H1) to (H5) hold with $A$ replaced by $A+H$.

Proof. This is obvious, except for the fact that condition (H4) holds if $\varepsilon>0$ is small enough. Let $t_{0} \in \overline{\mathbf{R}}$ be given. By (H3) and the continuity of $A$ and by the equivalence of the norm of $W$ with the graph norm of $A\left(t_{0}\right)$ (Theorem 2.1 (iii)), there are an open interval ${ }^{3} J_{t_{0}}$ about $t_{0}$ and $\beta>0$ such that $\left\|A(t) x+H(t) x-A\left(t_{0}\right) x\right\|_{X} \leq$ $2 \varepsilon\left\|A\left(t_{0}\right) x\right\|_{X}+\beta\|x\|_{X}$ for all $t \in J_{t_{0}}$ and all $x \in W$. It now follows from Theorem 2.3 with $A_{0}=A\left(t_{0}\right)$ that (H4) holds with $A$ replaced by $A+H$ and $t \in J_{t_{0}}$ if $\varepsilon>0$ is small enough. Thus, by the compactness of $\overline{\mathbf{R}}$, (H4) holds with $A$ replaced by $A+H$ and all $t \in \overline{\mathbf{R}}$ after shrinking $\varepsilon>0$ if necessary.

In Theorem 2.2, $A_{0}$ may also be viewed in $\mathcal{L}(W, X)$ if $W=D\left(A_{0}\right)$ is equipped with the graph norm, and then $R\left(i \xi, A_{0}\right) \in \mathcal{L}(X, W)$ whenever $i \xi \notin \sigma\left(A_{0}\right)$. The following theorem shows that condition (ii) of Theorem 2.2 can also be formulated in this (different) setting.

TheOrem 2.5. Let $X$ be a Banach space and let $A_{0}$ be an unbounded operator on $X$ with domain $D\left(A_{0}\right)=W$ equipped with the graph norm of $A_{0}$. Then, condition (ii) of Theorem 2.2 holds if and only if there is a constant $C_{0}>0$ such that, for some $n_{0} \in \mathbf{N}$,

$$
r_{\mathcal{L}(X, W)}\left(\left\{R\left(i 2^{n} a, A_{0}\right): n \geq n_{0}\right\}\right) \leq C_{0}, \quad \forall a \in \mathbf{R}, 1 \leq|a| \leq 2 .
$$

Likewise, the boundedness of $\left\|\xi R\left(i \xi, A_{0}\right)\right\|_{\mathcal{L}(X)}$ and of $\left\|R\left(i \xi, A_{0}\right)\right\|_{\mathcal{L}(X, W)}$ for $|\xi| \geq$ $r_{0}$ and some $r_{0}>0$ are equivalent.

Proof. We only prove the equivalence of (2.1) and (2.5) since the equivalence of the boundedness of $\left\|\xi R\left(i \xi, A_{0}\right)\right\|_{\mathcal{L}(X)}$ and of $\left\|R\left(i \xi, A_{0}\right)\right\|_{\mathcal{L}(X, W)}$ for $|\xi| \geq r_{0}$ is entirely similar and technically simpler.

Suppose that (2.5) holds. Given $\kappa \in \mathbf{N}$ and $n \in \mathbf{N}$ and $x_{k} \in X, 1 \leq k \leq \kappa$, we have

$$
i 2^{n_{k}} a R\left(i 2^{n_{k}} a, A_{0}\right) x_{k}=x_{k}+A_{0} R\left(i 2^{n_{k}} a, A_{0}\right) x_{k},
$$

for every $a \in \mathbf{R}$ with $1 \leq|a| \leq 2$, so that

$$
\begin{array}{r}
\int_{0}^{1}\left\|\sum_{k=1}^{\kappa} r_{k}(\tau) 2^{n_{k}} a R\left(i 2^{n_{k}} a, A_{0}\right) x_{k}\right\|_{X} d \tau \leq \int_{0}^{1}\left\|\sum_{k=1}^{\kappa} r_{k}(\tau) x_{k}\right\|_{X} d \tau+ \\
\int_{0}^{1}\left\|A_{0}\left(\sum_{k=1}^{\kappa} r_{k}(\tau) R\left(i 2^{n_{k}} a, A_{0}\right) x_{k}\right)\right\|_{X} d \tau .
\end{array}
$$

Since $W$ is equipped with the graph norm of $A_{0}$,

$$
\left\|A_{0}\left(\sum_{k=1}^{\kappa} r_{k}(\tau) R\left(i 2^{n_{k}} a, A_{0}\right) x_{k}\right)\right\|_{X} \leq\left\|\sum_{k=1}^{\kappa} r_{k}(\tau) R\left(i 2^{n_{k}} a, A_{0}\right) x_{k}\right\|_{W}
$$

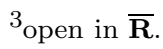


and hence it follows from (2.5) that

$$
\int_{0}^{1}\left\|A_{0}\left(\sum_{k=1}^{\kappa} r_{k}(\tau) R\left(i 2^{n_{k}} a, A_{0}\right) x_{k}\right)\right\|_{X} d \tau \leq C_{0} \int_{0}^{1}\left\|\sum_{k=1}^{\kappa} r_{k}(\tau) x_{k}\right\|_{X} d \tau .
$$

if $n_{k} \geq n_{0}$. By substitution into (2.7), we obtain

$$
\int_{0}^{1}\left\|\sum_{k=1}^{\kappa} r_{k}(\tau) 2^{n_{k}} a R\left(i 2^{n_{k}} a, A_{0}\right) x_{k}\right\|_{X} d \tau \leq\left(C_{0}+1\right) \int_{0}^{1}\left\|\sum_{k=1}^{\kappa} r_{k}(\tau) x_{k}\right\|_{X} d \tau,
$$

so that (2.1) holds.

Conversely, suppose that (2.1) holds. Rewrite (2.6) in the form

$$
i 2^{n_{k}} a R\left(i 2^{n_{k}} a, A_{0}\right) x_{k}-x_{k}=A_{0} R\left(i 2^{n_{k}} a, A_{0}-\lambda_{0} I\right) x_{k} .
$$

This yields

$$
\begin{aligned}
& \int_{0}^{1}\left\|A_{0}\left(\sum_{k=1}^{\kappa} r_{k}(\tau) R\left(i 2^{n_{k}} a, A_{0}\right) x_{k}\right)\right\|_{X} d \tau \leq \\
& \int_{0}^{1}\left\|\sum_{k=1}^{\kappa} r_{k}(\tau) 2^{n_{k}} a R\left(i 2^{n_{k}} a, A_{0}\right) x_{k}\right\|_{X} d \tau+\int_{0}^{1}\left\|\sum_{k=1}^{\kappa} r_{k}(\tau) x_{k}\right\|_{X} d \tau,
\end{aligned}
$$

and hence, with $C_{0}$ and $n_{0}$ from (2.1),

$$
\int_{0}^{1}\left\|A_{0}\left(\sum_{k=1}^{\kappa} r_{k}(\tau) R\left(i 2^{n_{k}} a, A_{0}\right) x_{k}\right)\right\|_{X} d \tau \leq\left(C_{0}+1\right) \int_{0}^{1}\left\|\sum_{k=1}^{\kappa} r_{k}(\tau) x_{k}\right\|_{X} d \tau
$$

if $n_{k} \geq n_{0}$.

On the other hand, since $|a| \geq 1$ and $n_{0} \in \mathbf{N}$, then

$$
\left\{R\left(i 2^{n} a, A_{0}\right): n \geq n_{0}\right\} \subset[0,1]\left\{2^{n} a R\left(i 2^{n} a, A_{0}\right): n \geq n_{0}\right\},
$$

where the set in the right-hand side consists of the products $\alpha 2^{n} a R\left(i 2^{n} a, A_{0}\right)$ for some $\alpha \in[0,1]$ and some $n \geq n_{0}$. Hence (for instance by $[\mathbf{3 2}]$ ),

$$
r_{\mathcal{L}(X)}\left(\left\{R\left(i 2^{n} a, A_{0}\right): n \geq n_{0}\right\}\right) \leq r_{\mathcal{L}(X)}\left(\left\{2^{n} a R\left(i 2^{n} a, A_{0}\right): n \geq n_{0}\right\}\right)
$$

so that $r_{\mathcal{L}(X)}\left(\left\{R\left(i 2^{n} a, A_{0}\right): n \geq n_{0}\right\}\right) \leq C_{0}$, irrespective of $1 \leq|a| \leq 2$. Thus, if $n_{k} \geq n_{0}$

$$
\int_{0}^{1}\left\|\sum_{k=1}^{\kappa} r_{k}(\tau) R\left(i 2^{n_{k}} a, A_{0}\right) x_{k}\right\|_{X} d \tau \leq C_{0} \int_{0}^{1}\left\|\sum_{k=1}^{\kappa} r_{k}(\tau) x_{k}\right\|_{X} d \tau .
$$

Together with (2.8) and since the norm of $W$ is the graph norm of $A_{0}$, we obtain

$$
\int_{0}^{1}\left\|\sum_{k=1}^{\kappa} r_{k}(\tau) R\left(i 2^{n_{k}} a, A_{0}\right) x_{k}\right\|_{W} d \tau \leq\left(2 C_{0}+1\right) \int_{0}^{1}\left\|\sum_{k=1}^{\kappa} r_{k}(\tau) x_{k}\right\|_{X} d \tau .
$$

so that (2.5) holds.

The next result is a special case of [32], using the fact that Banach spaces with UMD have nontrivial Rademacher type $([\mathbf{3 2}])$. It is the r-bounded version of the elementary result that a subset of $\mathcal{L}(X, Y)$ is bounded if and only if the subset of the corresponding adjoints is bounded in $\mathcal{L}\left(Y^{*}, X^{*}\right)$. 
Theorem 2.6. Let $X$ and $Y$ be Banach spaces with UMD. Given $\mathcal{T} \subset \mathcal{L}(X, Y)$, denote by $\mathcal{T}^{*}$ the set $\mathcal{T}^{*}:=\left\{T^{*}: T \in \mathcal{T}\right\} \subset \mathcal{L}\left(Y^{*}, X^{*}\right)$. Then, $\mathcal{T}$ is r-bounded if and only if $\mathcal{T}^{*}$ is r-bounded. Furthermore, there are constants $c(X)>0$ and $c^{*}(Y)>0$ depending only upon $X$ and $Y$, respectively, such that $r_{\mathcal{L}\left(Y^{*}, X^{*}\right)}\left(\mathcal{T}^{*}\right) \leq$ $c(X) r_{\mathcal{L}(X, Y)}(\mathcal{T})$ and that $r_{\mathcal{L}(X, Y)}(\mathcal{T}) \leq c^{*}(Y) r_{\mathcal{L}\left(Y^{*}, X^{*}\right)}\left(\mathcal{T}^{*}\right)$.

\section{The semi-Fredholm property}

This section is devoted to proving Theorem 3.1 below, a first and main step towards the proof of Theorem 1.1.

THEOREM 3.1. Let $X$ be a Banach space with UMD. If the family $(A(t))_{t \in \mathbf{R}}$ of unbounded operators on $X$ satisfies the assumptions (H1) to (H5), then for every $p \in(1, \infty)$ the operator $D_{A}:=\frac{d}{d t}-A$ is semi-Fredholm with index $\nu \in \mathbf{Z} \cup\{-\infty\}$ from $W^{1, p}(\mathbf{R}, X) \cap L^{p}(\mathbf{R}, W)$ to $L^{p}(\mathbf{R}, X)$. (In other words, $D_{A}$ has closed range and a finite dimensional null space.)

From now on, with $X$ and $W$ as in Theorem 3.1, it will be convenient to use the notation

$$
\mathcal{X}^{p}:=L^{p}(\mathbf{R}, X), \quad \mathcal{W}^{p}:=W^{1, p}(\mathbf{R}, X) \cap L^{p}(\mathbf{R}, W)
$$

and we shall assume throughout this section that $(A(t))_{t \in \mathbf{R}} \subset \mathcal{L}(W, X)$ satisfies the assumptions (H1) to (H5) without further mention. Also, $p \in(1, \infty)$ is chosen once and for all.

We need a series of lemmas, notably about the operators $D_{A(s)-\lambda I}$ when both $s$ and $\lambda$ are parameters and hence $D_{A(s)-\lambda I}=\frac{d}{d t}-(A(s)-\lambda I)$ acts on functions $u=u(t)$.

Lemma 3.2. (i) Let $s_{0} \in \mathbf{R}$ be given and let $\lambda_{0} \in \mathbf{R}$ be such that $\sigma\left(A\left(s_{0}\right)-\lambda_{0} I\right) \cap$ $i \mathbf{R}=\emptyset$. Then, there are an open interval $J_{0}$ about $s_{0}$ and a constant $C\left(s_{0}\right)>0$ such that $D_{A(s)-\lambda_{0} I} \in G L\left(\mathcal{W}^{p}, \mathcal{X}^{p}\right)$ for every $s \in J_{0}$ and that $\left\|D_{A(s)-\lambda_{0} I}^{-1}\right\|_{\mathcal{L}\left(\mathcal{X}^{p}, \mathcal{W}^{p}\right)} \leq$ $C\left(s_{0}\right)$ for every $s \in J_{0}$.

(ii) There is $T>0$ such that $D_{A(s)} \in G L\left(\mathcal{W}^{p}, \mathcal{X}^{p}\right)$ for every $s \in(-\infty,-T) \cup(T, \infty)$ and there is a constant $C_{\infty}>0$ such that $\left\|D_{A(s)}^{-1}\right\|_{\mathcal{L}\left(\mathcal{X}^{p}, \mathcal{W}^{p}\right)} \leq C_{\infty}$ for every $s \in$ $(-\infty,-T) \cup(T, \infty)$.

Proof. (i) By Theorem 2.1 (iii), the operator $A\left(s_{0}\right)$ is a closed unbounded operator on $X$ with domain $W$ equipped with a norm equivalent to the graph norm of $A\left(s_{0}\right)$, also the graph norm of $A\left(s_{0}\right)-\lambda_{0} I$. Next, by (H4), $A\left(s_{0}\right)$ satisfies the condition (2.3) of Theorem 2.3 and $-\lambda_{0} I$ is $A\left(s_{0}\right)$-bounded with relative bound 0 . Thus, by Theorem 2.3, $A\left(s_{0}\right)-\lambda_{0} I$ also satisfies (2.3), i.e., condition (ii) of Theorem 2.2. Since $W=D\left(A\left(s_{0}\right)-\lambda_{0} I\right)$ is dense in $X$ by (H1) and since $\sigma\left(A\left(s_{0}\right)-\lambda_{0} I\right) \cap i \mathbf{R}=$ $\emptyset$, it follows from that theorem that $D_{A\left(s_{0}\right)-\lambda_{0} I} \in G L\left(\mathcal{W}^{p}, \mathcal{X}^{p}\right)$.

It is readily checked that (H2) implies that the mapping $s \in \mathbf{R} \mapsto D_{A(s)-\lambda_{0} I} \in$ $\mathcal{L}\left(\mathcal{W}^{p}, \mathcal{X}^{p}\right)$ is continuous. By the openness of $G L\left(\mathcal{W}^{p}, \mathcal{X}^{p}\right)$ in $\mathcal{L}\left(\mathcal{W}^{p}, \mathcal{X}^{p}\right)$, it thus follows that $D_{A(s)-\lambda_{0} I} \in G L\left(\mathcal{W}^{p}, \mathcal{X}^{p}\right)$ with $\left\|D_{A(s)-\lambda_{0} I}^{-1}\right\|_{\mathcal{L}\left(\mathcal{X}^{p}, \mathcal{W}^{p}\right)}$ bounded by a constant $C\left(s_{0}\right)>0$ if $s \in J_{0}$ and $J_{0}$ is a small enough open interval about $s_{0}$. This proves (i).

(ii) The procedure is similar, just replacing $A\left(s_{0}\right)$ by $A_{ \pm}$and using (H3) instead of (H2). The assumption (H5) ensures that Theorem 2.2 is available with $A_{0}=$ $A_{ \pm}$. 
Lemma 3.3. There are a finite set $\Lambda \subset \mathbf{R}$ and a constant $C>0$ such that, for every $s \in \mathbf{R}$, the following two properties hold:

(i) There is $\lambda_{0} \in \Lambda$ such that $D_{A(s)-\lambda_{0} I} \in G L\left(\mathcal{W}^{p}, \mathcal{X}^{p}\right)$ and $\left\|D_{A(s)-\lambda_{0} I}^{-1}\right\|_{\mathcal{L}\left(\mathcal{X}^{p}, \mathcal{W}^{p}\right)} \leq$ C.

(ii) There is $T>0$ such that (i) holds with $\lambda_{0}=0$ whenever $|s|>T$, that is, $D_{A(s)} \in G L\left(\mathcal{W}^{p}, \mathcal{X}^{p}\right)$ and $\left\|D_{A(s)}^{-1}\right\|_{\mathcal{L}\left(\mathcal{X}^{p}, \mathcal{W}^{p}\right)} \leq C$ if $|s|>T$.

Proof. Let $T>0$ be as in part (ii) of Lemma 3.2 and let $s_{0} \in[-T, T]$ be given. From Theorem 2.1 (ii), $\sigma\left(A\left(s_{0}\right)\right)$ is discrete. As a result, there is $\lambda_{0} \in \mathbf{R}$ such that $\sigma\left(A\left(s_{0}\right)-\lambda_{0} I\right) \cap i \mathbf{R}=\emptyset$ and Lemma 3.2 (i) shows that there are an open interval $J_{0}$ about $s_{0}$ and a constant $C\left(s_{0}\right)>0$ such that $D_{A(s)-\lambda_{0} I} \in G L\left(\mathcal{W}^{p}, \mathcal{X}^{p}\right)$ and $\left\|D_{A(s)-\lambda_{0} I}^{-1}\right\|_{\mathcal{L}\left(\mathcal{X}^{p}, \mathcal{W}^{p}\right)} \leq C\left(s_{0}\right)$ if $s \in J_{0}$.

Cover $[-T, T]$ by finitely many such open intervals $J_{\ell}$ about $s_{\ell} \in[-T, T], 1 \leq$ $\ell \leq N$, with corresponding $\lambda_{\ell} \in \mathbf{R}$ and set $\Lambda:=\left\{0, \lambda_{1}, \ldots, \lambda_{N}\right\}$. If $s \in \mathbf{R}$, we have either $s \in[-T, T]$ or $s \in(-\infty,-T) \cup(T, \infty)$. In the first case, $s \in J_{\ell}$ for some $\ell \in$ $\{1, \ldots, N\}$, whence $D_{A(s)-\lambda_{\ell} I}^{-1} \in G L\left(\mathcal{W}^{p}, \mathcal{X}^{p}\right)$ and $\left\|D_{A(s)-\lambda_{\ell} I}^{-1}\right\|_{\mathcal{L}\left(\mathcal{X}^{p}, \mathcal{W}^{p}\right)} \leq C\left(s_{\ell}\right)$.

In the second case, $D_{A(s)} \in G L\left(\mathcal{W}^{p}, \mathcal{X}^{p}\right)$ and $\left\|D_{A(s)}^{-1}\right\|_{\mathcal{L}\left(\mathcal{X}^{p}, \mathcal{W}^{p}\right)} \leq C_{\infty}$ by part (ii) of Lemma 3.2. Thus, $C=\max \left\{C_{\infty}, \max _{1 \leq i \leq N} C\left(s_{i}\right)\right\}$ works in (i) and (ii).

Lemma 3.4. There is $\varepsilon>0$ such that, for every $\theta \in C^{\infty}(\mathbf{R})$ and every $u \in \mathcal{W}^{p}$,

$$
\begin{aligned}
& \sup _{s, t \in \operatorname{Supp} \theta}\|A(s)-A(t)\|_{\mathcal{L}(W, X)} \leq \varepsilon \Rightarrow \\
& \|\theta u\|_{\mathcal{W}^{p}} \leq \varepsilon^{-1}\left(\left\|\theta D_{A} u\right\|_{\mathcal{X}^{p}}+\left\|u \frac{d \theta}{d t}\right\|_{\mathcal{X}^{p}}+\|\theta u\|_{\mathcal{X}^{p}}\right) .
\end{aligned}
$$

Furthermore, there is $T>0$ such that whenever Supp $\theta \subset(-\infty,-T) \cup(T, \infty)$, then

$$
\|\theta u\|_{\mathcal{W}^{p}} \leq \varepsilon^{-1}\left(\left\|\theta D_{A} u\right\|_{\mathcal{X}^{p}}+\left\|u \frac{d \theta}{d t}\right\|_{\mathcal{X}^{p}}\right) .
$$

Proof. Let $u \in \mathcal{W}^{p}$ be given and set $f:=D_{A} u$. The multiplication of both sides by $\theta \in C^{\infty}(\mathbf{R})$ yields $D_{A}(\theta u)=u \frac{d \theta}{d t}+\theta f$. Pick $s_{0} \in S u p p \theta$ and let $\lambda_{0} \in \Lambda$ be given by Lemma 3.3 (i). Then,

$$
D_{A\left(s_{0}\right)-\lambda_{0} I}(\theta u)=\left(A-A\left(s_{0}\right)\right) \theta u+\theta f+u \frac{d \theta}{d t}+\lambda_{0} \theta u
$$

and hence, by Lemma 3.3 (i),

$$
\|\theta u\|_{\mathcal{W}^{p}} \leq C\left(\left\|\left(A-A\left(s_{0}\right)\right) \theta u\right\|_{\mathcal{X}^{p}}+\|\theta f\|_{\mathcal{X}^{p}}+\left\|u \frac{d \theta}{d t}\right\|_{\mathcal{X}^{p}}+\left|\lambda_{0}\right||| \theta u \|_{\mathcal{X}^{p}}\right),
$$

where $C>0$ is a constant independent of $s_{0}, u$ and $\theta$. By writing

$$
\left\|\left(A-A\left(s_{0}\right)\right) \theta u\right\|_{\mathcal{X}^{p}}=\left(\int_{\operatorname{Supp} \theta}\left\|\left(A(t)-A\left(s_{0}\right)\right) \theta(t) u(t)\right\|_{X}^{p} d t\right)^{\frac{1}{p}},
$$


we obtain the estimate

$$
\begin{aligned}
& \left\|\left(A-A\left(s_{0}\right)\right) \theta u\right\|_{\mathcal{X}^{p}} \leq \sup _{t \in S u p p \theta}\left\|A(t)-A\left(s_{0}\right)\right\|_{\mathcal{L}(W, X)}\|\theta u\|_{L^{p}(\mathbf{R}, W)} \leq \\
& \sup _{s, t \in \operatorname{Supp} \theta}\|A(s)-A(t)\|_{\mathcal{L}(W, X)}\|\theta u\|_{\mathcal{W}^{p}} .
\end{aligned}
$$

Since $\Lambda$ is finite, we now may change $C$ into $C \max \left\{1, \max _{\lambda \in \Lambda}|\lambda|\right\}$ in (3.4) to get

$$
\begin{aligned}
&\|\theta u\|_{\mathcal{W}^{p}} \leq C \sup _{s, t \in S u p p} \|\|A(s)-A(t)\|_{\mathcal{L}(W, X)}\|\theta u\|_{\mathcal{W}^{p}}+ \\
& C\left(\|\theta f\|_{\mathcal{X}^{p}}+\left\|u \frac{d \theta}{d t}\right\|_{\mathcal{X}^{p}}+\|\theta u\|_{\mathcal{X}^{p}}\right),
\end{aligned}
$$

which yields (3.2) with $\varepsilon=\frac{1}{2 C}$ independent of $u$ and $\theta$ since $f:=D_{A} u$.

The proof of (3.3) is similar, using part (ii) instead of part (i) of Lemma 3.3, which shows that we may choose $\lambda_{0}=0$ in (3.4) if $T>0$ is large enough and Supp $\theta \subset(-\infty,-T) \cup(T, \infty)$. In particular, by $(\mathrm{H} 3), T$ can be chosen so that $\|A(s)-A(t)\|_{\mathcal{L}(W, X)} \leq \frac{1}{2 C}$ whenever $s, t \geq T$ or $s, t \leq-T$. This yields (3.3) with $\varepsilon=\frac{1}{2 C}$ when $\operatorname{Supp} \theta \subset(-\infty,-T)$ or when $\operatorname{Supp} \theta \subset(T, \infty)$ and hence when $\operatorname{Supp} \theta \subset(-\infty,-T) \cup(T, \infty)$.

The last lemma follows readily from the uniform continuity of $A$ on compact intervals.

LEMMA 3.5. Given $\varepsilon>0$ and $T>0$, there is a covering of $[-T, T]$ by finitely many bounded open intervals $J_{\ell}, 1 \leq \ell \leq M$, such that $\sup _{s, t \in J_{\ell}} \| A(s)-$ $A(t) \|_{\mathcal{L}(W, X)} \leq \varepsilon$ for every $1 \leq \ell \leq M$.

Proof of Theorem 3.1. By Yood's criterion ([14, p. 78] ), it suffices to show that $D_{A}$ is proper on the closed bounded subsets of $\mathcal{W}^{p}$, i.e., that every bounded sequence $\left(u_{n}\right) \subset \mathcal{W}^{p}$ such that

$$
D_{A} u_{n} \rightarrow f \text { in } \mathcal{X}^{p}
$$

contains a convergent subsequence.

Let $\varepsilon>0$ be given by Lemma 3.4 and let $T>0$ be such that (3.3) holds. With this choice of $\varepsilon$ and $T$, Lemma 3.5 yields finitely many bounded open intervals $J_{1}, \ldots, J_{M}$ covering $[-T, T]$ such that

$$
\sup _{s, t \in J_{\ell}}\|A(s)-A(t)\|_{\mathcal{L}(W, X)} \leq \varepsilon, \quad 1 \leq \ell \leq M
$$

Set $J_{0}:=(-\infty, T)$ and $J_{M+1}:=(T, \infty)$, so that $\mathbf{R}=\cup_{\ell=0}^{M+1} J_{\ell}$ and let $\left(\theta_{\ell}\right)_{0 \leq \ell \leq M+1}$ be a partition of unity subordinate to this covering. From the boundedness of $J_{\ell}$ when $1 \leq \ell \leq M$, we infer that $\theta_{0}(t)=1$ for $-t>0$ large enough and $\theta_{M+1}(t)=1$ for $t>0$ large enough. Thus, Supp $\frac{d \theta_{0}}{d t}$ and Supp $\frac{d \theta_{M+1}}{d t}$ are compact. Since also $\operatorname{Supp} \theta_{\ell} \subset J_{\ell}$ is compact when $1 \leq \ell \leq M$, there is $0<\tilde{T}<\infty$ such that

$$
\begin{aligned}
& \operatorname{Supp} \theta_{\ell} \subset(-\tilde{T}, \tilde{T}), \quad 1 \leq \ell \leq M, \\
& \qquad \operatorname{Supp} \frac{d \theta_{\ell}}{d t} \subset(-\tilde{T}, \tilde{T}), \quad 0 \leq \ell \leq M+1 .
\end{aligned}
$$


Since Supp $\theta_{0} \subset(T, \infty)$ and $\operatorname{Supp} \theta_{M+1} \subset(-\infty,-T)$ and due to the choice of $\varepsilon$ and $T$, it follows from (3.3) that

$$
\left\|\theta_{\ell} u\right\|_{\mathcal{W}^{p}} \leq \varepsilon^{-1}\left(\left\|\theta_{\ell} D_{A} u\right\|_{\mathcal{X}^{p}}+\left\|u \frac{d \theta_{\ell}}{d t}\right\|_{\mathcal{X}^{p}}\right), \quad \ell=0 \text { or } M+1,
$$

for every $u \in \mathcal{W}^{p}$. If now $1 \leq \ell \leq M$, we infer from ( 3.6) and $\operatorname{Supp} \theta_{\ell} \subset J_{\ell}$ that $\sup _{s, t \in \text { Supp }_{\ell}}\|A(s)-A(t)\|_{\mathcal{L}(W, X)} \leq \varepsilon$. Hence, by (3.2) with $\theta=\theta_{\ell}$,

$$
\left\|\theta_{\ell} u\right\|_{\mathcal{W}^{p}} \leq \varepsilon^{-1}\left(\left\|\theta_{\ell} D_{A} u\right\|_{\mathcal{X}^{p}}+\left\|u \frac{d \theta_{\ell}}{d t}\right\|_{\mathcal{X}^{p}}+\left\|\theta_{\ell} u\right\|_{\mathcal{X}^{p}}\right), \quad 1 \leq \ell \leq M
$$

for every $u \in \mathcal{W}^{p}$. By adding up (3.8) and (3.9),

$$
\sum_{\ell=0}^{M+1}\left\|\theta_{\ell} u\right\|_{\mathcal{W}^{p}} \leq \varepsilon^{-1}\left(\sum_{\ell=0}^{M+1}\left\|\theta_{\ell} D_{A} u\right\|_{\mathcal{X}^{p}}+\sum_{\ell=0}^{M+1}\left\|u \frac{d \theta_{\ell}}{d t}\right\|_{\mathcal{X}^{p}}+\sum_{\ell=1}^{M}\left\|\theta_{\ell} u\right\|_{\mathcal{X}^{p}}\right) .
$$

We shall set

$$
\mathcal{X}_{\tilde{T}}^{p}:=L^{p}((-\tilde{T}, \tilde{T}), X), \quad \mathcal{W}_{\tilde{T}}^{p}:=W^{1, p}((-\tilde{T}, \tilde{T}), X) \cap L^{p}((-\tilde{T}, \tilde{T}), W) .
$$

By (3.7) and (3.10) and $\sum_{\ell=0}^{M+1} \theta_{\ell}=1$ (whence $u=\sum_{\ell=0}^{M+1} \theta_{\ell} u$ ), there is a constant $C>0$ independent of $u$ such that

$$
\|u\|_{\mathcal{W}^{p}} \leq C\left(\left\|D_{A} u\right\|_{\mathcal{X}^{p}}+\|u\|_{\mathcal{X}_{\tilde{T}}^{p}}\right), \quad u \in \mathcal{W}^{p} .
$$

Since the embedding $W \hookrightarrow X$ is compact and the interval $(-\tilde{T}, \tilde{T})$ is bounded, it follows from Simon [37, Theorem 1] that the embedding $\mathcal{W}_{\tilde{T}}^{p} \hookrightarrow \mathcal{X}_{\tilde{T}}^{p}$ is compact. We may thus assume with no loss of generality that the sequence $\left(u_{n}\right)$ (bounded in $\mathcal{W}^{p}$ and hence in $\left.\mathcal{W}_{\tilde{T}}^{p}\right)$ is convergent in $\mathcal{X}_{\tilde{T}}^{p}$. Then, by letting $u=u_{n}-u_{m}$ in (3.12) and since $\left(D_{A} u_{n}\right)$ is convergent in $\mathcal{X}^{p}$ by $(3.5)$, we find that $\left(u_{n}\right)$ is convergent in $\mathcal{W}^{p}$. This completes the proof.

Remark 3.1. When A satisfies the hypotheses of Theorem 3.1, it follows from (3.12) that the boundedness of $u$ and $D_{A} u$ in $\mathcal{X}^{p}$ implies the boundedness of $u$ in $\mathcal{W}^{p}$.

\section{Regularity and the Fredholm property}

We continue to assume that $X$ is a Banach space with UMD and that $p \in$ $(1, \infty)$. With $p^{\prime} \in(1, \infty)$ denoting the Hölder conjugate of $p$, we introduce the spaces

$$
\mathcal{W}_{*}^{p^{\prime}}:=L^{p^{\prime}}\left(\mathbf{R}, W^{*}\right), \quad \mathcal{X}_{*}^{p^{\prime}}:=W^{1, p^{\prime}}\left(\mathbf{R}, W^{*}\right) \cap L^{p^{\prime}}\left(\mathbf{R}, X^{*}\right) .
$$

The spaces $\mathcal{W}_{*}^{p^{\prime}}$ and $\mathcal{X}_{*}^{p^{\prime}}$ correspond to the space $\mathcal{X}^{p}$ and $\mathcal{W}^{p}$ in (3.1) (in that order), upon replacing $X, W$ and $p$ by $W^{*}, X^{*}$ and $p^{\prime}$, respectively. It is important that

$$
L^{p^{\prime}}\left(\mathbf{R}, X^{*}\right)=\left(L^{p}(\mathbf{R}, X)\right)^{*}=\left(\mathcal{X}^{p}\right)^{*} .
$$

For this, see Edwards $[\mathbf{2 0}]$ and recall that Banach spaces with UMD are reflexive (see the Introduction). In fact, if $\langle\langle\cdot, \cdot\rangle\rangle$ denotes the duality pairing between $\mathcal{X}^{p}$ and $\left(\mathcal{X}^{p}\right)^{*}$, then

$$
\left\langle\left\langle v, v^{*}\right\rangle\right\rangle:=\int_{\mathbf{R}}\left\langle u(t), v^{*}(t)\right\rangle_{X, X^{*}} d t, \quad v \in \mathcal{X}^{p}, v^{*} \in\left(\mathcal{X}^{p}\right)^{*}=L^{p^{\prime}}\left(\mathbf{R}, X^{*}\right),
$$

where $\langle\cdot, \cdot\rangle_{X, X^{*}}$ is the duality pairing between $X$ and $X^{*}$. 
With

$$
\left(\operatorname{rge} D_{A}\right)^{\perp}:=\left\{v^{*} \in\left(\mathcal{X}^{p}\right)^{*}:\left\langle\left\langle v, v^{*}\right\rangle\right\rangle=0, \forall v \in \operatorname{rge} D_{A}\right\},
$$

we first prove that if $(A(t))_{t \in \mathbf{R}}$ satisfies (H1) to (H5), then

$$
\left(\text { rge } D_{A}\right)^{\perp}=\operatorname{ker} D_{-A^{*}},
$$

where $D_{A}: \mathcal{W}^{p} \rightarrow \mathcal{X}^{p}$ and $D_{-A^{*}}=\frac{d}{d t}+A^{*}: \mathcal{X}_{*}^{p^{\prime}} \rightarrow \mathcal{W}_{*}^{p^{\prime}}$. By (H2) and (H3) and duality, $A^{*}: \mathbf{R} \rightarrow \mathcal{L}\left(X^{*}, W^{*}\right)$ is continuous and bounded, whence $D_{-A^{*}}$ does map $\mathcal{X}_{*}^{p^{\prime}}$ to $\mathcal{W}_{*}^{p^{\prime}}$ and (4.5) makes sense since $\mathcal{X}_{*}^{p^{\prime}} \subset\left(\mathcal{X}^{p}\right)^{*}$ by (4.1) and (4.2). Of course, $\left(\operatorname{rge} D_{A}\right)^{\perp}=\operatorname{ker}\left(D_{A}\right)^{*}$ with $\left(D_{A}\right)^{*} \in \mathcal{L}\left(\left(\mathcal{X}^{p}\right)^{*},\left(\mathcal{W}^{p}\right)^{*}\right)$ the adjoint of $D_{A}$. But $\left(D_{A}\right)^{*}$ has no convenient explicit characterization and hence a direct proof that $\operatorname{dim} \operatorname{ker}\left(D_{A}\right)^{*}<\infty$ is not available. On the other hand, (4.5) shows that (rge $\left.D_{A}\right)^{\perp}$, a subspace of $\left(\mathcal{X}^{p}\right)^{*}$, is actually a subspace of $\mathcal{X}_{*}^{p^{\prime}} \subset\left(\mathcal{X}^{p}\right)^{*}$, so that $(4.5)$ is a "regularity" result. We now prove it in

Lemma 4.1. Let $X$ be a Banach space and let $(A(t))_{t \in \mathbf{R}}$ be a family of unbounded operators on $X$ satisfying the assumptions (H1) to (H5). Then, $v^{*} \in\left(\mathcal{X}^{p}\right)^{*}$ and

$$
\left\langle\left\langle D_{A} u, v^{*}\right\rangle\right\rangle=0,
$$

for every $u \in \mathcal{W}^{p}$ if and only if $v^{*} \in \mathcal{X}_{*}^{p^{\prime}}$ and $D_{-A^{*}} v^{*}=0$.

Proof. Suppose first that $v^{*} \in\left(\mathcal{X}^{p}\right)^{*}=L^{p^{\prime}}\left(\mathbf{R}, X^{*}\right)$ and that $\left\langle\left\langle D_{A} u, v^{*}\right\rangle\right\rangle=0$. Since the embedding $W \hookrightarrow X$ is continuous, every $x^{*} \in X^{*}$ is in $W^{*}$ and the restriction of $x^{*}$ to $W$ (dense in $X$ ) determines $x^{*}$ uniquely. Thus,

$$
\left\langle x, x^{*}\right\rangle_{X, X^{*}}=\left\langle x, x^{*}\right\rangle_{W, W^{*}}, \quad \forall x \in W, \quad \forall x^{*} \in X^{*} \subset W^{*} .
$$

In particular, the embedding $X^{*} \subset W^{*}$ is continuous, so that $L^{p^{\prime}}\left(\mathbf{R}, X^{*}\right) \subset L^{p^{\prime}}\left(\mathbf{R}, W^{*}\right)$ and hence $v^{*} \in L^{p^{\prime}}\left(\mathbf{R}, W^{*}\right)$.

It remains to prove that $\frac{d v^{*}}{d t} \in L^{p^{\prime}}\left(\mathbf{R}, W^{*}\right)$ and that $D_{-A^{*}} v^{*}=0$ as distributions with values in $W^{*}$. The former property follows from the latter because $D_{-A^{*}} v^{*}=0$ amounts to $\frac{d v^{*}}{d t}=-A^{*} v^{*} \in L^{p^{\prime}}\left(\mathbf{R}, W^{*}\right)$ since $A^{*}: \mathbf{R} \rightarrow \mathcal{L}\left(X^{*}, W^{*}\right)$ is continuous and bounded (by (H2), (H3) and duality) and $v^{*} \in L^{p^{\prime}}\left(\mathbf{R}, X^{*}\right)$.

Let $x \in W \subset X$ and $\varphi \in \mathcal{D}(\mathbf{R})=C_{0}^{\infty}(\mathbf{R})$ be given, so that $\varphi x \in \mathcal{W}^{p}$ and hence $\left\langle\left\langle D_{A}(\varphi x), v^{*}\right\rangle\right\rangle=\int_{\mathbf{R}}\left\langle\frac{d \varphi}{d t}(t) x-\varphi(t) A(t) x, v^{*}(t)\right\rangle_{X, X^{*}} d t=0$ by (4.3) and (4.6). This may be rewritten as

$$
\int_{\mathbf{R}}\left\langle x, \frac{d \varphi}{d t}(t) v^{*}(t)\right\rangle_{X, X^{*}}-\int_{\mathbf{R}}\left\langle x, \varphi(t) A^{*}(t) v^{*}(t)\right\rangle_{W, W^{*}} d t=0
$$

Since the Bochner integral commutes with duality pairings, this is also

$$
\left\langle x, \int_{\mathbf{R}} \frac{d \varphi}{d t}(t) v^{*}(t) d t\right\rangle_{X, X^{*}}-\left\langle x, \int_{\mathbf{R}} \varphi(t) A^{*}(t) v^{*}(t) d t\right\rangle_{W, W^{*}}=0 .
$$

It follows from (4.7) and the assumption $x \in W$ that the pairing $\langle\cdot, \cdot\rangle_{X, X^{*}}$ in (4.8) may be replaced by $\langle\cdot, \cdot\rangle_{W, W^{*}}$. In this form, (4.8) expresses that

$$
\int_{\mathbf{R}} \frac{d \varphi}{d t}(t) v^{*}(t) d t-\int_{\mathbf{R}} \varphi(t) A^{*}(t) v^{*}(t) d t=0,
$$

in $W^{*}$. By definition of the derivative in the sense of distributions and since $\varphi \in$ $\mathcal{D}(\mathbf{R})$ is arbitrary, this is just $D_{-} A^{*} v^{*}=0$ as distributions with values in $W^{*}$. 
Conversely, suppose that $v^{*} \in \mathcal{X}_{*}^{p^{\prime}}$ and $D_{-A^{*}} v^{*}=0$. Then, $v^{*} \in\left(\mathcal{X}^{p}\right)^{*}$ by (4.1) and (4.2) and (4.9) holds for every $\varphi \in \mathcal{D}(\mathbf{R})$. By reversing the above steps, it follows that $\left\langle\left\langle D_{A}(\varphi x), v^{*}\right\rangle\right\rangle=0$ for every $x \in W$ and every $\varphi \in \mathcal{D}(\mathbf{R})$. Thus, by linearity, $\left\langle\left\langle D_{A} u, v^{*}\right\rangle\right\rangle=0$ for $u \in \mathcal{D}(\mathbf{R}) \otimes W$ and hence for $u \in \mathcal{W}^{p}$ by a straightforward density argument (since $W$ is dense in $X$ ).

Theorem 4.2. Let $X$ be a Banach space with $U M D$ and let $(A(t))_{t \in \mathbf{R}}$ be a family of unbounded operators on $X$ satisfying the assumptions (H1) to (H5). Then, the operator $D_{A}$ is Fredholm from $\mathcal{W}^{p}$ to $\mathcal{X}^{p}$ for every $p \in(1, \infty)$. Furthermore, $D_{-A^{*}}$ is Fredholm from $\mathcal{X}_{*}^{p^{\prime}}$ to $\mathcal{W}_{*}^{p^{\prime}}$ and in this setting, index $D_{-A^{*}}=-$ index $D_{A}$ and $\left(\text { rge } D_{A}\right)^{\perp}=\operatorname{ker} D_{-A^{*}}$.

Proof. By Theorem 3.1, $D_{A}$ is semi-Fredholm with $\operatorname{dim} \operatorname{ker} D_{A}<\infty$. To prove that $D_{A}$ is Fredholm, it suffices to show that $\operatorname{dim} \operatorname{ker} D_{-A^{*}}<\infty$ when $D_{-A^{*}}$ acts from $\mathcal{X}_{*}^{p^{\prime}}$ to $\mathcal{W}_{*}^{p^{\prime}}$ : If so, codim rge $D_{A}=\operatorname{dim} \operatorname{ker} D_{-A^{*}}$ by Lemma 4.1 and the closedness of rge $D_{A}$. The characterization $\left(\operatorname{rge} D_{A}\right)^{\perp}=\operatorname{ker} D_{-A^{*}}$ also follows from Lemma 4.1.

We shall prove the stronger result that $D_{-A^{*}}$ is semi-Fredholm with finite dimensional null space from $\mathcal{X}_{*}^{p^{\prime}}$ to $\mathcal{W}_{*}^{p^{\prime}}$. To do this, we check that we may replace $A$ by $A^{*}$ in Theorem 3.1, so that $X$ becomes $W^{*}$ and $W$ becomes $X^{*}$. To begin with, $W$ is a Banach space with UMD by Theorem 2.1 (iv), whence $W^{*}$ is a Banach space with UMD. For convenience, in the remainder of the proof, we call $\left(\mathrm{H}^{*} 1\right), \ldots$, $\left(\mathrm{H}^{*} 5\right)$ the conditions $(\mathrm{H} 1), \ldots,(\mathrm{H} 5)$ for $A^{*}$.

As noted in the proof of Lemma 4.1, $X^{*} \hookrightarrow W^{*}$ (continuous embedding). Let $j$ denote the embedding $W \hookrightarrow X$, so that $j$ is compact by (H1). Then, $j^{*} \in$ $\mathcal{L}\left(X^{*}, W^{*}\right)$ is compact and, for $x^{*} \in X^{*}, j^{*} x^{*} \in W^{*}$ is the restriction of $x^{*}$ to $W$. Thus, $j^{*}$ is the (compact) embedding $X^{*} \hookrightarrow W^{*}$. Since $X$ and $W$ are reflexive (Theorem 2.1 (iv)), we have $\left(j^{*}\right)^{*}=j$ and, since $j$ is one to one, it follows that $j^{*}\left(X^{*}\right)=X^{*}$ is dense in $W^{*}$. This proves $\left(\mathrm{H}^{*} 1\right)$. From now on, we simply denote by " $I$ " (identity) the embeddings $j$ and $j^{*}$.

The conditions $\left(\mathrm{H}^{*} 2\right)$ and $\left(\mathrm{H}^{*} 3\right)$ (with $A_{ \pm}$replaced by $\left.A_{ \pm}^{*}\right)$ are trivial from (H2) and (H3) and duality.

Given $t \in \overline{\mathbf{R}}$, the property $\left(\mathrm{H}^{*} 4\right)$ requires the existence of $C_{0}^{*}(t)>0$ and of $n_{0}^{*}(t) \in \mathbf{N}$ such that

$$
r_{\mathcal{L}\left(W^{*}\right)}\left(\left\{2^{n} a R\left(i 2^{n} a, A^{*}(t)\right): n \geq n_{0}^{*}(t)\right\}\right) \leq C_{0}^{*}(t), \quad a \in \mathbf{R}, 1 \leq|a| \leq 2,
$$

where $A^{*}(t)$ is viewed as an unbounded operator on $W^{*}$ with domain $X^{*}$. This inequality follows from (H4) and Theorems 2.5 and 2.6. Specifically, by (H4) and Theorem 2.5 there are $C_{0}(t)>0$ and $n_{0}(t) \in \mathbf{N}$ such that

$$
r_{\mathcal{L}(X, W)}\left(\left\{R\left(i 2^{n} a, A(t)\right): n \geq n_{0}(t)\right\}\right) \leq C_{0}(t), \quad a \in \mathbf{R}, 1 \leq|a| \leq 2 .
$$

The embedding $X^{*} \hookrightarrow W^{*}$ being the adjoint of the embedding $W \hookrightarrow X$, we have $(\lambda I-A)^{*}=\lambda I-A^{*}$ and hence $R\left(\lambda, A^{*}(t)\right)=R(\lambda, A(t))^{*}$. Thus,

$$
r_{\mathcal{L}\left(W^{*}, X^{*}\right)}\left(\left\{R\left(i 2^{n} a, A^{*}(t)\right): n \geq n_{0}(t)\right\}\right) \leq c(X) C_{0}(t),
$$

by (4.10) and Theorem 2.6, where $c(X)>0$ depends only upon $X$. Thus, $\left(\mathrm{H}^{*} 4\right)$ holds by another application of Theorem 2.5, provided that we check that the norm of $X^{*}$ is equivalent to the graph norm of $A^{*}(t)$. This is done below.

By the continuity of the embedding $X^{*} \hookrightarrow W^{*}$, the graph norm of $A^{*}(t)$ is equivalent to the graph norm of $A^{*}(t)-\lambda I=(A(t)-\lambda I)^{*}$ for every $\lambda \in \mathbf{C}$. On 
the other hand, since $\sigma(A(t)) \neq \mathbf{C}$, there is $\lambda$ such that $A(t)-\lambda I \in G L(W, X)$ (see Theorem 2.1 (i) and (iii)). As a result, it is not restrictive to prove the equivalence of the norm of $X^{*}$ with the graph norm of $A^{*}(t)$ under the additional assumption that $A(t) \in G L(W, X)$.

Given $x^{*} \in X^{*}$ and $x \in X$, we have

$$
\begin{array}{r}
\left|\left\langle x, x^{*}\right\rangle_{X, X^{*}}\right|=\left|\left\langle A(t) A^{-1}(t) x, x^{*}\right\rangle_{X, X^{*}}\right|=\left|\left\langle A^{-1}(t) x, A^{*}(t) x^{*}\right\rangle_{W, W^{*}}\right| \leq \\
\left\|A^{-1}(t) x\right\|\left\|_{W}\right\| A^{*}(t) x^{*} \|_{W^{*}},
\end{array}
$$

so that

$$
\begin{aligned}
\left\|x^{*}\right\|_{X^{*}} \leq\left\|A^{-1}(t)\right\|_{\mathcal{L}(X, W)}\left\|A^{*}(t) x^{*}\right\|_{W^{*}} \leq \\
\left\|A^{-1}(t)\right\|_{\mathcal{L}(X, W)}\left(\left\|x^{*}\right\|_{W^{*}}+\left\|A^{*}(t) x^{*}\right\|_{W^{*}}\right) .
\end{aligned}
$$

Conversely, $\left\|x^{*}\right\|_{W^{*}}+\left\|A^{*}(t) x^{*}\right\|_{W^{*}} \leq\left(C+\left\|A^{*}(t)\right\|_{\mathcal{L}\left(X^{*}, W^{*}\right)}\right)\left\|x^{*}\right\|_{X^{*}}$ where $C>0$ is the norm of the embedding $X^{*} \hookrightarrow W^{*}$. This proves the desired equivalence of norms and completes the proof of $\left(\mathrm{H}^{*} 4\right)$.

The last condition, $\left(\mathrm{H}^{*} 5\right)$, is trivial from (H5) and Theorem 2.1 (i).

At this stage, we have that $D_{-A^{*}}$ is semi-Fredholm with finite dimensional null space from $\mathcal{X}_{*}^{p^{\prime}}$ to $\mathcal{W}_{*}^{p^{\prime}}$ by Theorem 3.1 for $-A^{*}$. As noted at the beginning of the proof, this yields codim rge $D_{A}=\operatorname{dim} \operatorname{ker} D_{-A^{*}}$ and hence

$$
\text { index } D_{A}=\operatorname{dim} \operatorname{ker} D_{A}-\operatorname{dim} \operatorname{ker} D_{-A^{*}} \text {. }
$$

In turn, if $A$ is replaced by $-A^{*}$ in this identity (a substitution justified by the first part of the proof), then $-A^{*}$ is replaced by $A^{* *}$. But $A^{* *}=A$ since $X$ and $W$ are reflexive. This gives index $D_{-A^{*}}=\operatorname{dim} \operatorname{ker} D_{-A^{*}}-\operatorname{dim} \operatorname{ker} D_{A}=-i n d e x D_{A}$ and the proof is complete.

Theorem 4.2 raises the question of the $p$-independence of the index of $D_{A}$, which will be proved in Theorem 5.1.

REMARK 4.1. It follows at once from Theorem 4.2 and the local constancy of the index of Fredholm operators that the index of $D_{A}$ is a homotopy invariant of the paths $(A(t))_{t \in \mathbf{R}}$ satisfying the assumptions (H1) to (H5).

\section{Elementary properties of the index of $D_{A}$}

We begin with a regularity result that also proves the $p$-independence of the index of $D_{A}$ in Theorem 4.2.

TheOrem 5.1. Let $X$ be a Banach space with $U M D$ and let the family $(A(t))_{t \in \mathbf{R}}$ of unbounded operators on $X$ satisfy the assumptions (H1) to (H5). If $p, q \in(1, \infty)$ and $f \in \mathcal{X}^{p} \cap \mathcal{X}^{q}$ and if $u \in \mathcal{W}^{p}$ satisfies $D_{A} u=f$, then $u \in \mathcal{W}^{p} \cap \mathcal{W}^{q}$. In particular, the index of $D_{A}: \mathcal{W}^{p} \rightarrow \mathcal{X}^{p}$ is independent of $p \in(1, \infty)$.

Proof. We shall use a localization argument based on the fact that when $A(t)=A_{0}$ is constant and $\sigma\left(A_{0}\right) \cap i \mathbf{R}=\emptyset$, then $D_{A_{0}} \in G L\left(\mathcal{W}^{p}, \mathcal{X}^{p}\right) \cap G L\left(\mathcal{W}^{q}, \mathcal{X}^{q}\right) \cap$ $G L\left(\mathcal{W}^{p} \cap \mathcal{W}^{q}, \mathcal{X}^{p} \cap \mathcal{X}^{q}\right)([\mathbf{3 2}])$.

Given $T>0$ and $\theta \in \mathcal{D}(\overline{\mathbf{R}})$ with support in $(T, \infty)$ satisfying $\theta=1$ in $[T+1, \infty)$, we have $D_{A}(\theta u)=\theta f+\frac{d \theta}{d t} u$ and $D_{A}(\theta u)=D_{A_{T}}(\theta u)$ where $A_{T}(t)=A(T)$ if $t<T$ and $A_{T}(t)=A(t)$ if $t \geq T$. Thus, $D_{A_{T}}(\theta u)=\theta f+\frac{d \theta}{d t} u$. By increasing $T, D_{A_{T}}$ is an arbitrarily small perturbation of $D_{A_{+}}$and since $\sigma\left(A_{+}\right) \cap i \mathbf{R}=\emptyset$ by (H5), it 
follows that $D_{A_{T}} \in G L\left(\mathcal{W}^{p}, \mathcal{X}^{p}\right) \cap G L\left(\mathcal{W}^{q}, \mathcal{X}^{q}\right) \cap G L\left(\mathcal{W}^{p} \cap \mathcal{W}^{q}, \mathcal{X}^{p} \cap \mathcal{X}^{q}\right)$ for $T$ large enough. Since $u \in \mathcal{W}^{p} \subset C^{0}(\mathbf{R}, X)$ and $\frac{d \theta}{d t} u$ has compact support (whence $\left.\theta f, \frac{d \theta}{d t} u \in \mathcal{X}^{p} \cap \mathcal{X}^{q}\right)$, this implies that $\theta u \in \mathcal{W}^{p} \cap \mathcal{W}^{q}$. Likewise, $\theta u \in \mathcal{W}^{p} \cap \mathcal{W}^{q}$ if $\theta \in \mathcal{D}(\overline{\mathbf{R}})$ has support in $(-\infty,-T)$ with $T>0$ large enough and $\theta=1$ in $(-\infty,-T-1]$.

Let now $t_{0} \in \mathbf{R}$ be fixed. Choose $\lambda_{0} \in \mathbf{R}$ such that $\sigma\left(A\left(t_{0}\right)-\lambda_{0} I\right) \cap i \mathbf{R}=\emptyset$ (see Theorem 2.1 (ii)), so that $D_{A\left(t_{0}\right)-\lambda_{0} \mathrm{I}} \in G L\left(\mathcal{W}^{p}, \mathcal{X}^{p}\right) \cap G L\left(\mathcal{W}^{q}, \mathcal{X}^{q}\right) \cap G L\left(\mathcal{W}^{p} \cap \mathcal{W}^{q}\right.$, $\left.\mathcal{X}^{p} \cap \mathcal{X}^{q}\right)$. Given $\varepsilon>0$, define $A_{t_{0}, \varepsilon}(t)=A\left(t_{0}-\varepsilon\right)$ if $t<t_{0}-\varepsilon, A_{t_{0}, \varepsilon}(t)=A\left(t_{0}+\varepsilon\right)$ if $t>t_{0}+\varepsilon$ and $A_{t_{0}, \varepsilon}(t)=A(t)$ if $t \in[t-\varepsilon, t+\varepsilon]$. Then, $D_{A_{t_{0}, \varepsilon}-\lambda_{0} \mathrm{I}}$ is arbitrarily close to $D_{A\left(t_{0}\right)-\lambda_{0} \mathrm{I}}$ if $\varepsilon>0$ is small enough, whence $D_{A_{t_{0}, \varepsilon}-\lambda_{0} \mathrm{I}} \in G L\left(\mathcal{W}^{p}, \mathcal{X}^{p}\right) \cap$ $G L\left(\mathcal{W}^{q}, \mathcal{X}^{q}\right) \cap G L\left(\mathcal{W}^{p} \cap \mathcal{W}^{q}, \mathcal{X}^{p} \cap \mathcal{X}^{q}\right)$. Next, $D_{A}(\theta u)=D_{A-\lambda_{0} \mathrm{I}}(\theta u)+\lambda_{0} \theta u=$ $D_{A_{t_{0}, \varepsilon}-\lambda_{0} \mathrm{I}}(\theta u)+\lambda_{0} \theta u$ if $\theta \in \mathcal{D}(\mathbf{R})$ has support in $\left(t_{0}-\varepsilon, t_{0}+\varepsilon\right)$. Thus, $D_{A_{t_{0}, \varepsilon}-\lambda_{0} \mathrm{I}}(\theta u)=$ $\theta f+\frac{d \theta}{d t} u-\lambda_{0} \theta u \in \mathcal{X}^{p} \cap \mathcal{X}^{q}$ and hence $\theta u \in \mathcal{W}^{p} \cap \mathcal{W}^{q}$ from the above.

That $u \in \mathcal{W}^{p} \cap \mathcal{W}^{q}$ follows from the results of the previous two paragraphs via a partition of unity similar to the one used in the proof of Theorem 3.1. We skip the (routine) details.

With the choice $f=0$, we obtain that the null space of $D_{A}: \mathcal{W}^{p} \rightarrow \mathcal{X}^{p}$ is contained in $\mathcal{W}^{p} \cap \mathcal{W}^{q} \subset \mathcal{W}^{q}$ for all $1<q<\infty$ and hence independent of $p$. By replacing $A$ by $-A^{*}$ (see the proof of Theorem 4.2 ), the null space of $D_{-A^{*}}: \mathcal{X}_{*}^{p^{\prime}} \rightarrow$ $\mathcal{W}_{*}^{p^{\prime}}$ is also independent of $p$ and hence index $D_{A}$ is independent of $p$ by (4.11).

In our next result, we give a simple condition ensuring that $D_{A}$ has index 0 . It will be used for the general characterization of the index in the next section.

THEOREM 5.2. Let $X$ be a Banach space with UMD and let the family $(A(t))_{t \in \mathbf{R}}$ of unbounded operators on $X$ satisfy the assumptions (H1) to (H5). If also $\sigma(A(t)) \cap$ $i \mathbf{R}=\emptyset$ for every $t \in \mathbf{R}$, then the operator $D_{A}:=\frac{d}{d t}-A$ is Fredholm of index 0 from $\mathcal{W}^{p}$ to $\mathcal{X}^{p}$ for every $p \in(1, \infty)$.

Proof. From Theorem 4.2, we already know that $D_{A}$ is Fredholm. For $T \geq 0$, let $A_{T}$ denote the operator $A_{T}(t)=A(t)$ for $t \in[-T, T], A_{T}(t)=A(-T)$ for $t<-T$ and $A_{T}(t)=A(T)$ for $t>T$. It is obvious that $A_{T}$ satisfies the assumptions (H1) to (H5), so that once again by Theorem 4.2 , the operator $D_{A_{T}}$ is Fredholm from $\mathcal{W}^{p}$ to $\mathcal{X}^{p}$. Since $D_{A_{T}} \in \mathcal{L}\left(\mathcal{W}^{p}, \mathcal{X}^{p}\right)$ depends continuously upon $T$, the index of $D_{A_{T}}$ is independent of $T \geq 0$. On the other hand, $\lim _{T \rightarrow \infty}\left\|D_{A_{T}}-D_{A}\right\|_{\mathcal{L}\left(\mathcal{W}^{p}, \mathcal{X}^{p}\right)}=0$ by (H3), so that index $D_{A_{T}}=$ index $D_{A}$ if $T>0$ is large enough. Thus, index $D_{A}=$ index $D_{A_{0}}$ from the above. But $\left(A_{0}(t)\right)_{t \in \mathbf{R}}$ is the constant family $A(0)$ and, since $\sigma(A(0)) \cap i \mathbf{R}=\emptyset$, it follows from Theorem 2.2 that $D_{A_{0}}$ is an isomorphism. As a result, index $D_{A}=0$.

Next, we examine the effect of perturbations of $A$ on index $D_{A}$. The proof of the following theorem is not based on the usual argument that compact perturbations of Fredholm operators preserve the index (see Remark 5.1).

THEOREM 5.3. Let $X$ be a Banach space with UMD and let the family $(A(t))_{t \in \mathbf{R}}$ of unbounded operators on $X$ satisfy the assumptions (H1) to (H5). Suppose that $K \in C^{0}(\mathbf{R}, \mathcal{K}(W, X))$ where $\mathcal{K}(W, X) \subset \mathcal{L}(W, X)$ is the subspace of compact operators and that $\lim _{|t| \rightarrow \infty}\|K(t)\|_{\mathcal{L}(W, X)}=0$. Then, the operator $D_{A+K}$ is Fredholm from $\mathcal{W}^{p}$ to $\mathcal{X}^{p}$ for every $p \in(1, \infty)$ and index $D_{A+K}=$ index $D_{A}$. 
Proof. It suffices to show that $A+K$ satisfies (H1) to (H5). If so, after replacing $K$ by $\alpha K$ for $\alpha \in[0,1]$, it follows from Theorem 4.2 that $D_{A+\alpha K}$ is Fredholm and then index $D_{A+K}=$ index $D_{A}$ by the local constancy of the index.

It is obvious that $A+K$ satisfies (H1), (H2), (H3) and (H5). We prove that $A+K$ satisfies (H4). Observe first that, by the equivalence of the norm of $W$ with the graph norm of $A(t)$ (Theorem 2.1 (iii)), the assumption that $C(t) \in \mathcal{K}(W, X)$ (with $C( \pm \infty)=0$ ) implies that $K(t)$ is $A(t)$-compact for $t \in \overline{\mathbf{R}}$ and hence $A(t)$ bounded with $A(t)$-bound 0 (Hess $[\mathbf{2 2}]$; recall that $X$ is reflexive). Therefore, (H4) for $A+K$ follows from (H4) for $A$ and Theorem 2.3.

Remark 5.1. In Theorem 5.3, $D_{A+K}$ need not be a compact perturbation of $D_{A}$, i.e., the multiplication operator $\widehat{K}: \mathcal{W}^{p} \rightarrow \mathcal{X}^{p}$ induced by $K$ in the obvious way need not be compact. In fact, from the given proof, Theorem 5.3 remains valid if the assumption $K(t) \in \mathcal{K}(W, X)$ is replaced by the condition that $K(t)$ is $A(t)$-bounded with $A(t)$-bound 0 . Thus, Theorem 5.3 does not follow from the classical stability properties of Fredholm operators. However, $\widehat{K}$ is compact when $X$ is a (separable) Hilbert space (see [34]). Also, $\widehat{K}$ is compact when $K \in C^{0}(\mathbf{R}, \mathcal{L}(X)$ ) (a special case of Theorem 5.3 since the embedding $W \hookrightarrow X$ is compact). If so, the compactness of $\widehat{K}$ can be derived from [37, Theorem 1] after replacing $K$ by a step function with values in $\mathcal{L}(X)$ and vanishing outside a compact interval. It is noteworthy that if $K \in C^{0}(\mathbf{R}, \mathcal{K}(X))$ and $A(t)=A_{0}$ is constant, then Theorem 5.3 is still true even if the embedding $D\left(A_{0}\right)=W \hookrightarrow X$ is not compact (see [32], or [1, Theorem B] when $X$ is a Hilbert space and $A_{0}$ is bounded).

As a simple corollary to Theorem 5.3, we obtain another sufficient condition for the index of $D_{A}$ to be 0 .

Corollary 5.4. Let $X$ be a Banach space with UMD and let the family $(A(t))_{t \in \mathbf{R}}$ of unbounded operators on $X$ satisfy the assumptions (H1) to (H5). Suppose also that $A_{-}=A_{+}$and that $A(t)-A_{+} \in \mathcal{K}(W, X)$ for every $t \in \mathbf{R}$. Then, the operator $D_{A}$ is Fredholm of index 0 from $\mathcal{W}^{p}$ to $\mathcal{X}^{p}$ for every $p \in(1, \infty)$.

Another sufficient condition for the index of $D_{A}$ to be 0 arises as a special case of Theorem 6.7 later.

REMARK 5.2. All the index theorems of this paper remain true if $X$ is a real space. This follows from a straightforward general property: If $E$ and $F$ are real Banach spaces and $L \in \mathcal{L}(E, F)$, denote by $E_{\mathbf{C}}, F_{\mathbf{C}}$ and $L_{\mathbf{C}} \in \mathcal{L}\left(E_{\mathbf{C}}, F_{\mathbf{C}}\right)$ their complexifications. Then, $L$ and $L_{\mathbf{C}}$ have closed range simultaneously, $\left(L_{\mathbf{C}}\right)^{*}=$ $\left(L^{*}\right)_{\mathbf{C}}$ and $\operatorname{dim} \operatorname{ker} L=\operatorname{dim}_{\mathbf{C}} \operatorname{ker} L_{\mathbf{C}}$, dim $\operatorname{ker} L^{*}=\operatorname{dim}_{\mathbf{C}} \operatorname{ker}\left(L_{\mathbf{C}}\right)^{*}$, so that the indices of $L$ and $L_{\mathbf{C}}$ are simultaneously defined and equal. This property is relevant here since passing from $X$ to $X_{\mathbf{C}}$ amounts to changing $\mathcal{W}^{p}, \mathcal{X}^{p}$ and $D_{A}$ into their complexifications.

\section{Calculation of the index of $D_{A}$}

In this section, we show that the index of $D_{A}$ coincides with the spectral flow of $A$, that is, with the algebraic count of the eigenvalues of $A(t)$ that cross the imaginary axis when $t$ varies from $-\infty$ to $\infty$. Of course, this characterization, which generalizes the one given in [34] in the Hilbert and selfadjoint case, makes sense only when the crossing of the imaginary axis can be defined without ambiguity. 
Let $X$ and $W$ be complex Banach spaces such that $W \subset X$ and the embedding $W \hookrightarrow X$ is compact. For the time being, we need not assume that $X$ has the UMD property. Every operator $L \in \mathcal{L}(W, X)$ can be viewed as an unbounded operator on $X$ with domain $W$ and hence has a well defined spectrum $\sigma(L)$. If $\sigma(L) \neq \mathbf{C}$, then $L$ has compact resolvent and $L-\lambda I \in G L(W, X)$ for every $\lambda \in \mathbf{C} \backslash \sigma(L)$ (see the proof of Theorem 2.1). We set

$$
\mathcal{O}:=\{L-\lambda I: L \in \mathcal{L}(W, X), \sigma(L) \neq \mathbf{C}, \lambda \in \mathbf{C}\}
$$

and, for $k \in \mathbf{N}$, we define $\mathcal{S}_{k} \subset \mathcal{O}$ by

$$
\mathcal{S}_{k}:=\left\{N \in \mathcal{O}: \operatorname{codim}_{\mathbf{C}} \text { rge } N=k\right\} .
$$

Lemma 6.1. (i) The subset $\mathcal{O}$ is an open subset of $\mathcal{L}(W, X)$.

(ii) $\mathcal{S}_{k}$ is a complex analytic submanifold of $\mathcal{O}$ of (complex) codimension $k^{2}$. Furthermore, the tangent space $T_{N} \mathcal{S}_{k}$ is given by

$$
T_{N} \mathcal{S}_{k}=\{H \in \mathcal{L}(W, X): H(\operatorname{ker} N) \subset \operatorname{rge} N\} .
$$

Proof. (i) The set of those $L \in \mathcal{L}(W, X)$ such that $\sigma(L) \neq \mathbf{C}$ is open in $\mathcal{L}(W, X)$ and hence, for every $\lambda \in \mathbf{C}$, the set $\mathcal{O}_{\lambda}=\{L-\lambda I: L \in \mathcal{L}(W, X), \sigma(L) \neq$ $\mathbf{C}\}$ is open in $\mathcal{L}(W, X)$. Thus, $\mathcal{O}=\cup_{\lambda \in \mathbf{C}} \mathcal{O}_{\lambda}$ is open in $\mathcal{L}(W, X)$.

(ii) First, note that $\mathcal{S}_{k}$ is also characterized by

$$
\mathcal{S}_{k}:=\left\{N \in \mathcal{O}: \operatorname{dim}_{\mathbf{C}} \operatorname{ker} N=k\right\} .
$$

Indeed, if $N \in \mathcal{S}_{k}$, then $N=L-\lambda I$ for some $L \in \mathcal{L}(W, X)$ such that $\sigma(L) \neq \mathbf{C}$ and some $\lambda \in \mathbf{C}$. Choose $\mu \in \mathbf{C} \backslash \sigma(L)$, so that $(L-\mu I)^{-1} \in \mathcal{L}(X, W)$ and hence $(L-\mu I)^{-1} \in \mathcal{L}(W)$ is compact. Since $N=(L-\mu I)\left(I+(\mu-\lambda)(L-\mu I)^{-1}\right)$, it follows that codim $\operatorname{rge}\left(I+(\mu-\lambda)(L-\mu I)^{-1}\right)=k$. Thus, $\operatorname{dim}_{\mathbf{C}} \operatorname{ker}\left(I+(\mu-\lambda)(L-\mu I)^{-1}\right)=k$ by the compactness of $(L-\mu I)^{-1} \in \mathcal{L}(W)$ and so $\operatorname{dim}_{\mathrm{C}} \operatorname{ker} N=k$. By reversing the above arguments, if $N \in \mathcal{O}$ and $\operatorname{dim}_{\mathbf{C}} \operatorname{ker} N=k$, then $N \in \mathcal{S}_{k}$. This proves (6.4).

Now, let $N_{0} \in \mathcal{S}_{k}$ and let $N \in \mathcal{O}$ be given. Let $P \in \mathcal{L}(X)$ denote a projection onto a complement of rge $N_{0}$ (recall that, in Banach spaces, bounded linear operators with finite corank have closed ranges, hence closed and complemented ranges) so that $Q:=I-P$ projects onto rge $N_{0}$. Let $\pi \in \mathcal{L}(W)$ denote a projection onto $\operatorname{ker} N_{0}$ with null-space $V:=\operatorname{ker} \pi$. If $\left\|N-N_{0}\right\|_{\mathcal{L}(W, X)}$ is small enough, then $\left(Q N_{\mid V}\right)^{-1} \in \mathcal{L}\left(\operatorname{rge} N_{0}, V\right)$ is well defined and $x \in \operatorname{ker} N$ if and only if $[(I-\pi) x=$ $-\left(Q N_{\mid V}\right)^{-1} Q N \pi x$ AND] $P N\left(I-\left(Q N_{\mid V}\right)^{-1} Q N\right) \pi x=0$ Thus, dim ker $N=k$ (and then $N \in \mathcal{S}_{k}$ by (6.4)) if and only if $P N_{\mid \operatorname{ker} N_{0}}-P N\left(Q N_{\mid V}\right)^{-1} Q N_{\mid \operatorname{ker} N_{0}}=0$. The left-hand side is a complex analytic function $F=F(N)$ from $\mathcal{O}$ to $\mathcal{L}\left(\operatorname{ker} N_{0}\right.$, rge $P$ ) with (surjective) derivative $D F\left(N_{0}\right) H=P H_{\mid \text {ker } N_{0}}(H \in \mathcal{L}(W, X))$. It follows that, locally near $N_{0}, \mathcal{S}_{k}$ coincides with $F^{-1}(0)$, a submanifold of $\mathcal{O}$ of complex codimension $k^{2}$, and that $T_{N_{0}} \mathcal{S}_{k}=\operatorname{ker} D F\left(N_{0}\right)=\left\{H \in \mathcal{L}(W, X): P H_{\mid \operatorname{ker} N_{0}}=0\right\}=$ $\left\{H \in \mathcal{L}(W, X): H\left(\operatorname{ker} N_{0}\right) \subset \operatorname{rge} N_{0}\right\}$. This completes the proof.

Given $A_{+}, A_{-} \in \mathcal{L}(W, X)$, denote by $\mathcal{M}\left(A_{+}, A_{-}\right)$the set of $C^{1}$ paths $A: \mathbf{R} \rightarrow$ $\mathcal{L}(W, X)$ such that $\lim _{t \rightarrow \pm \infty} A(t)=A_{ \pm}$and $\lim _{t \rightarrow \pm \infty} \dot{A}(t)=0$. It is obvious that $\mathcal{M}\left(A_{+}, A_{-}\right)$is a closed affine subspace of the space of $C^{1}$ paths $\mathbf{R} \rightarrow \mathcal{L}(W, X)$ bounded along with their derivatives, equipped with the norm

$$
\|\| A\|\|:=\sup _{t \in \mathbf{R}}\|A(t)\|_{\mathcal{L}(W, X)}+\sup _{t \in \mathbf{R}}\|\dot{A}(t)\|_{\mathcal{L}(W, X)} .
$$


In particular, $\mathcal{M}\left(A_{+}, A_{-}\right)$is a manifold with tangent space $\mathcal{M}(0,0)$ at each point. We denote by $\mathcal{U}\left(A_{+}, A_{-}\right)$the subset of $\mathcal{M}\left(A_{+}, A_{-}\right)$of those paths $A$ for which there are constants $C_{0}(t)>0$ and $r_{0}(t)>0$ such that

$$
\|R(i \xi, A(t))\|_{\mathcal{L}(X, W)} \leq C_{0}(t), \quad \xi \in \mathbf{R},|\xi| \geq r_{0}(t), \quad t \in \overline{\mathbf{R}} .
$$

Remark 6.1. By the continuity of $A$ on $\overline{\mathbf{R}}$ and the compactness of $\overline{\mathbf{R}}$, the constants $C_{0}(t)$ and $r_{0}(t)$ in (6.6) can be chosen independent of $t \in \overline{\mathbf{R}}$.

Lemma 6.2. (i) $\mathcal{U}\left(A_{+}, A_{-}\right)$is an open subset of $\mathcal{M}\left(A_{+}, A_{-}\right)$.

(ii) Given $A \in \mathcal{U}\left(A_{+}, A_{-}\right)$and $\varepsilon>0$, there is a path $B \in \mathcal{U}\left(A_{+}, A_{-}\right)$such that ${ }^{4}$ $\left|\left\|B-A|\||<\varepsilon\right.\right.$ and such that the mapping $(t, \xi) \in \mathbf{R}^{2} \mapsto B(t)-i \xi I \in \mathcal{O}$ intersects all the manifolds $\mathcal{S}_{k}$ transversely.

(iii) Assume $\sigma\left(A_{ \pm}\right) \cap i \mathbf{R}=\emptyset$. If $A \in \mathcal{U}\left(A_{+}, A_{-}\right)$and the mapping $(t, \xi) \in \mathbf{R}^{2} \mapsto$ $A(t)-i \xi I \in \mathcal{O}$ intersects all the manifolds $\mathcal{S}_{k}$ transversely, then $\sigma(A(t)) \cap i \mathbf{R} \neq \emptyset$ for only finitely many distinct values of $t \in \mathbf{R}$. Furthermore, if $\sigma\left(A\left(t_{j}\right)\right) \cap i \mathbf{R} \neq \emptyset$ for $1 \leq j \leq m$ and distinct values $t_{j} \in \mathbf{R}$, the operator $A\left(t_{j}\right)$ has only a finite number of imaginary eigenvalues $i \xi_{j, 1}, \ldots, i \xi_{j, \ell_{j}}$. These eigenvalues are algebraically simple and, for $t$ near $t_{j}$, the spectrum of $A(t)$ near $\left\{i \xi_{j, 1}, \ldots, i \xi_{j, \ell_{j}}\right\}$ consists of $\ell_{j}$ curves $\lambda_{j, 1}(t), \ldots, \lambda_{j, \ell_{j}}(t)$ of class $C^{1}$ and satisfying $\lambda_{j, \ell}\left(t_{j}\right)=i \xi_{j, \ell}$ and $\operatorname{Re} \dot{\lambda}_{j, \ell}\left(t_{j}\right) \neq 0$ for $1 \leq \ell \leq \ell_{j}$ and $1 \leq j \leq m$.

Proof. (i) Let $A \in \mathcal{U}\left(A_{+}, A_{-}\right)$and let $t_{0} \in \overline{\mathbf{R}}$ be given. We use the notation of (6.6). If $H \in \mathcal{L}(W, X)$ satisfies $\|H\|_{\mathcal{L}(W, X)}<C_{0}^{-1}\left(t_{0}\right)$ and if $\xi \in \mathbf{R},|\xi| \geq r_{0}\left(t_{0}\right)$, then $A\left(t_{0}\right)+H-i \xi I$ is invertible and $\left\|R\left(i \xi, A\left(t_{0}\right)+H\right)\right\|_{\mathcal{L}(X, W)} \leq \frac{C_{0}\left(t_{0}\right)}{1-C_{0}\left(t_{0}\right) \mid\|H\|_{\mathcal{L}(W, X)}}$. Thus, $\left\|R\left(i \xi, A\left(t_{0}\right)+H\right)\right\|_{\mathcal{L}(X, W)} \leq 2 C_{0}\left(t_{0}\right)$ if $\|H\|_{\mathcal{L}(W, X)}<\frac{1}{2} C_{0}^{-1}\left(t_{0}\right)$. Together with the continuity of $A$ on $\overline{\mathbf{R}}$, this implies that there is an open interval $J_{t_{0}}$ about $t_{0}$ such that, if $B \in \mathcal{M}\left(A_{+}, A_{-}\right)$and $\sup _{t \in \mathbf{R}}\|B(t)-A(t)\|_{\mathcal{L}(W, X)}$ is small enough, then $\|R(i \xi, B(t))\|_{\mathcal{L}(X, W)} \leq 2 C_{0}\left(t_{0}\right)$ for all $t \in J_{t_{0}}$. By the compactness of $\overline{\mathbf{R}}$, it follows that $\|R(i \xi, B(t))\|_{\mathcal{L}(X, W)}$ is uniformly bounded for all $t \in \overline{\mathbf{R}}$ if $\sup _{t \in \mathbf{R}}\|B(t)-A(t)\|_{\mathcal{L}(W, X)}$ is small enough (and hence if $\|B-A\| \|$ in (6.5) is small enough), so that $B \in \mathcal{U}\left(A_{+}, A_{-}\right)$.

(ii) Let $F: \mathcal{U}\left(A_{+}, A_{-}\right) \times \mathbf{R}^{2} \rightarrow \mathcal{O}$ be defined by $F(A, t, \xi):=A(t)-i \xi I$. Then, $F$ is of class $C^{1}$ and $\frac{\partial F}{\partial A}(A, t, \xi) H=H(t)$ for all $H \in \mathcal{M}(0,0)$, so that $\frac{\partial F}{\partial A}(A, t, \xi)$ is onto $\mathcal{L}(W, X)$. As a result, $F$ is transversal to $\mathcal{S}_{k}$ for all $k \in \mathbf{N}$. Thus, by the parametric transversality theorem (see for instance [3, p. 232], or [4]), the set of paths $A \in \mathcal{U}\left(A_{+}, A_{-}\right)$such that the mapping $(t, \xi) \in \mathbf{R}^{2} \mapsto A(t)-i \xi I \in \mathcal{O}$ is transversal to $\mathcal{S}_{k}$ is residual in $\mathcal{U}\left(A_{+}, A_{-}\right)$. Since $\mathcal{U}\left(A_{+}, A_{-}\right)$is an open subset of the complete metric space $\mathcal{M}\left(A_{+}, A_{-}\right)$, the intersection of these residual subsets is dense in $\mathcal{U}\left(A_{+}, A_{-}\right)$. The existence of the path $B$ follows at once from this property.

(iii) If $A \in \mathcal{U}\left(A_{+}, A_{-}\right)$and the mapping $(t, \xi) \in \mathbf{R}^{2} \mapsto A(t)-i \xi I \in \mathcal{O}$ intersects all the manifolds $\mathcal{S}_{k}$ transversely, then $A(t)-i \xi I \notin \mathcal{S}_{k}$ for every $(t, \xi) \in \mathbf{R}^{2}$ whenever $k \geq 2$ since, by Lemma $6.1, \operatorname{codim}_{\mathbf{R}} \mathcal{S}_{k} \geq 8$ in this case.

Thus, if $A\left(t_{0}\right)-i \xi_{0} I \in \cup_{k} \mathcal{S}_{k}$, then $A\left(t_{0}\right)-i \xi_{0} I \in \mathcal{S}_{1}$ and hence, by (6.4), $i \xi_{0}$ is an eigenvalue of $A\left(t_{0}\right)$ with geometric multiplicity 1 . We claim that, in fact, $i \xi_{0}$ has algebraic multiplicity 1 . Indeed, since $\mathcal{S}_{1}$ has real codimension 2 by Lemma 6.1 , the transversality condition implies that the range of the derivative

\footnotetext{
${ }^{4}$ See $(6.5)$.
} 
$(s, \zeta) \in \mathbf{R}^{2} \mapsto s A\left(t_{0}\right)-i \zeta I$ is a direct complement of $T_{A\left(t_{0}\right)-i \xi_{0} I} \mathcal{S}_{1}$. By (6.3), this means that if $\operatorname{ker}\left(A\left(t_{0}\right)-i \xi_{0} I\right)=\operatorname{span}\left\{x_{0}\right\}$, then

$$
s \dot{A}\left(t_{0}\right) x_{0}-i \zeta x_{0} \in \operatorname{rge}\left(A\left(t_{0}\right)-i \xi_{0} I\right) \Leftrightarrow s=\zeta=0 .
$$

By letting $s=0$ and $\zeta=1$, we find that $i x_{0} \notin$ rge $\left(A\left(t_{0}\right)-i \xi_{0} I\right)$, whence $x_{0} \notin$ rge $\left(A\left(t_{0}\right)-i \xi_{0} I\right)$. This shows that $i \xi_{0}$ has algebraic multiplicity 1 .

The condition $x_{0} \notin$ rge $\left(A\left(t_{0}\right)-i \xi_{0} I\right)$ also means that if $x_{0}^{*} \in X^{*}$ and $\operatorname{ker} x_{0}^{*}=$ rge $\left(A\left(t_{0}\right)-i \xi_{0} I\right)$, then $x_{0}^{*}\left(x_{0}\right) \neq 0$. It follows from this remark and from the implicit function theorem that, for $t$ near $t_{0}$, the (unique) eigenvalue of $A(t)$ near $i \xi_{0}$ is given by a $C^{1}$ function $\lambda(t)$ such that $\lambda\left(t_{0}\right)=i \xi_{0}$. Furthermore, $\operatorname{ker}(A(t)-\lambda(t) I)=$ $\operatorname{span}\{x(t)\}$ where $x(t) \in W$ is a (nonunique) $C^{1}$ function of $t$ satisfying $x\left(t_{0}\right)=$ $x_{0}$. By differentiating $A(t) x(t)-\lambda(t) x(t)=0$, we find that $\dot{A}\left(t_{0}\right) x_{0}-\dot{\lambda}\left(t_{0}\right) x_{0}=$ $-\left(A\left(t_{0}\right)-i \xi_{0} I\right) \dot{x}\left(t_{0}\right) \in \operatorname{rge}\left(A\left(t_{0}\right)-i \xi_{0} I\right)$, whence $\operatorname{Re} \dot{\lambda}\left(t_{0}\right) \neq 0$ by $(6.7)$.

To complete the proof, it suffices to show that $A(t)-i \xi I \in \mathcal{S}_{1}$ cannot occur for more than finitely many pairs $(t, \xi) \in \mathbf{R}^{2}$. This follows from the fact that such pairs are isolated (by transversality) and compact (by Remark 6.1 and the assumption $\left.\sigma\left(A_{ \pm}\right) \cap i \mathbf{R}=\emptyset\right)$.

Under the conditions of Lemma 6.2 (iii), we define

$$
s f(A)=-\sum_{j=1}^{m} \sum_{\ell=1}^{\ell_{j}} \operatorname{sgn} \operatorname{Re} \dot{\lambda}_{j, \ell}\left(t_{j}\right)
$$

(spectral flow of $A$ ). In particular, $s f(A)=0$ if $\sigma(A(t)) \cap i \mathbf{R}=\emptyset$ for all $t \in \mathbf{R}$.

Remark 6.2. When $\operatorname{dim} X<\infty$ and $W=X$, a different definition of the spectral flow can be given, which is valid for merely continuous paths ([34, Section 2]). The two definitions coincide for "transversal" $C^{1}$ paths. Furthermore, index $D_{A}=\operatorname{sf}(A)$ when $D_{A}$ acts from $W^{1,2}(\mathbf{R}, X)$ to $L^{2}(\mathbf{R}, X)$. From the $p$ independence of index $D_{A}$ (Theorem 5.1), this relation still holds when $D_{A}$ acts from $W^{1, p}(\mathbf{R}, X)$ to $L^{p}(\mathbf{R}, X)$ and $p \in(1, \infty)$.

As in [34] in the selfadjoint case, the general proof that index $D_{A}=s f(A)$ will be based on a preliminary construction. The proof of $[\mathbf{3 4}]$ is actually incomplete and we also provide the details of the (important) missing step.

We assume that the path $A$ satisfies the conditions of Lemma 6.2 (iii) and use the notation of that lemma. We also assume that $m \geq 1$, that is, that $\sigma(A(t)) \cap i \mathbf{R} \neq$ $\emptyset$ for at least one value $t \in \mathbf{R}$. Set

$$
Z_{m}:=\oplus_{\ell=1}^{\ell_{m}} \operatorname{ker}\left(A\left(t_{m}\right)-i \xi_{m, \ell} I\right) \subset W,
$$

so that $\operatorname{dim} Z_{m}=\ell_{m}$. Since each eigenvalue $i \xi_{m, \ell}$ is algebraically simple, it follows that $X=Z_{m} \oplus X_{m}$ where $X_{m}:=\cap_{\ell=1}^{\ell_{m}}$ rge $\left(A\left(t_{m}\right)-i \xi_{m, \ell} I\right)$ and hence that $W=$ $Z_{m} \oplus W_{m}$ where $W_{m}:=X_{m} \cap W$. Clearly, $A\left(t_{m}\right)-i \xi_{m, \ell} I$ is one-to-one on $W_{m}$ and maps $W_{m}$ onto $X_{m}$, The latter is readily checked from the definition of $X_{m}$ and the decompositions $W=Z_{m} \oplus W_{m}$ and $X=Z_{m} \oplus X_{m}$. For $\xi \notin\left\{\xi_{m, 1}, \ldots, \xi_{m, \ell_{m}}\right\}$, $A\left(t_{m}\right)-i \xi I$ is an isomorphism of $W$ onto $X$ and hence of $Z_{m}$ onto $Z_{m}$ and of $W_{m}$ onto $X_{m}$. Thus, $A\left(t_{m}\right)-i \xi I$ is an isomorphism of $W_{m}$ onto $X_{m}$ for all $\xi \in$ R. Furthermore, by $(6.6),\left\|\left(\left(A\left(t_{m}\right)-i \xi I\right)_{\mid W_{m}}\right)^{-1}\right\|_{\mathcal{L}\left(X_{m}, W_{m}\right)}$ is bounded for large enough $|\xi|$ and hence for all $\xi \in \mathbf{R}$ by continuity, say

$$
\left\|\left(\left(A\left(t_{m}\right)-i \xi I\right)_{\mid W_{m}}\right)^{-1}\right\|_{\mathcal{L}\left(X_{m}, W_{m}\right)} \leq C, \quad \xi \in \mathbf{R} .
$$


In the proof of Lemma 6.2 (iii), it was observed that $\operatorname{ker}\left(A(t)-\lambda_{m, \ell}(t) I\right)=$ $\operatorname{span}\left\{x_{\ell}(t)\right\}$, where $x_{\ell}(t) \in W$ is a (nonunique) $C^{1}$ function of $t$ near $t_{m}$. Set

$$
Z_{m}(t):=\oplus_{\ell=1}^{\ell_{m}} \operatorname{ker}\left(A(t)-\lambda_{m, \ell}(t) I\right)=\operatorname{span}\left\{x_{1}(t), \ldots, x_{\ell_{m}}(t)\right\}
$$

so that $Z_{m}$ in (6.9) is $Z_{m}\left(t_{m}\right)$. We claim that

(6.12) $W=Z_{m}(t) \oplus W_{m}$ and $(A(t)-i \xi I)\left(W_{m}\right) \cap Z_{m}(t)=\{0\}, \quad t \in J_{t_{m}}, \quad \xi \in \mathbf{R}$.

where $J_{t_{m}}$ is an open interval about $t_{m}$. Indeed, for $t$ close enough to $t_{m}$, we have $W=Z_{m}(t) \oplus W_{m}$ and $X=Z_{m}(t) \oplus X_{m}$ and the projection $P(t)$ onto $X_{m}$ corresponding to the latter splitting is bounded in norm. In addition, by (6.10), the operator $\left(A(t)-A\left(t_{m}\right)\right)\left(\left(A\left(t_{m}\right)-i \xi I\right)_{\mid W_{m}}\right)^{-1} \in \mathcal{L}\left(X_{m}\right)$ has arbitrarily small norm uniformly in $\xi \in \mathbf{R}$. It follows that the image of $X_{m}$ under $I+(A(t)-$ $\left.A\left(t_{m}\right)\right)\left(\left(A\left(t_{m}\right)-i \xi I\right)_{\mid W_{m}}\right)^{-1}$ intersects $Z_{m}(t)$ only at $\{0\}$ irrespective of $\xi \in \mathbf{R}$. Thus, $(A(t)-i \xi I)\left(W_{m}\right) \cap Z_{m}(t)=\{0\}$.

Above, we may further restrict $J_{t_{m}}$ so that the functions $\lambda_{m, \ell}(t)$ are defined and $C^{1}$ on $J_{t_{m}}$. Moreover, since $\lambda_{m, \ell}(t) \notin i \mathbf{R}$ for $0<\left|t-t_{m}\right|$ small enough by the condition $\operatorname{Re} \dot{\lambda}_{m, \ell}\left(t_{m}\right) \neq 0$, we may assume with no loss of generality that $\lambda_{m, \ell}(t) \notin i \mathbf{R}$ for $t \in \bar{J}_{t_{m}} \backslash\left\{t_{m}\right\}$ and $1 \leq \ell \leq \ell_{m}$. From now on, the functions $\lambda_{m, \ell}$ are extended to all of $\mathbf{R}$ as $C^{1}$ functions eventually constant and satisfying ${ }^{5}$

$$
\lambda_{m, \ell}(t) \notin i \mathbf{R}, \quad t \in \mathbf{R} \backslash\left\{t_{m}\right\}, \quad 1 \leq \ell \leq \ell_{m} .
$$

Let $u_{1}^{*}, \ldots, u_{\ell_{m}}^{*} \in W^{*}$ be such that $W_{m}=\cap_{\ell=1}^{\ell_{m}} \operatorname{ker} u_{\ell}^{*}$ and that $\left\langle x_{j}\left(t_{m}\right), u_{\ell}^{*}\right\rangle=\delta_{\ell j}$ (Kronecker delta). For $t$ close enough to $t_{m}$, say $t \in J_{t_{m}}$ after shrinking once again $J_{t_{m}}$ if necessary, and given $1 \leq k \leq \ell_{m}$, the system $\sum_{\ell=1}^{\ell_{m}} \alpha_{\ell k}\left\langle x_{j}(t), u_{\ell}^{*}\right\rangle=$ $\delta_{j k}, 1 \leq j \leq \ell_{m}$, has a unique solution $\left(\alpha_{1 k}(t), \ldots \alpha_{\ell_{m} k}(t)\right) \in \mathbf{C}^{\ell_{m}}$ and $x_{k}^{*}(t):=$ $\sum_{\ell=1}^{\ell_{m}} \alpha_{\ell k}(t) u_{\ell}^{*} \in W^{*}$ satisfies

$$
\left\langle x_{j}(t), x_{k}^{*}(t)\right\rangle=\delta_{k j} .
$$

Note that $x_{j}^{*}$ above is a $C^{1}$ function of $t \in J_{t_{m}}$. After possibly shrinking $J_{t_{m}}$ a last time, we can manage so that

$$
t_{1}, \ldots, t_{m-1} \in \mathbf{R} \backslash \bar{J}_{t_{m}} .
$$

Let $M(t) \in \mathcal{L}\left(\mathbf{C}^{\ell_{m}}\right)$ be the $C^{1}$ diagonal path defined by

$$
M(t):=\operatorname{diag}\left(-\bar{\lambda}_{m, 1}(t), \ldots,-\bar{\lambda}_{m, \ell_{m}}(t)\right) .
$$

Since the functions $\lambda_{m, \ell}$ have been extended so as to be eventually constant, $M$ is of class $C^{1}, \lim _{t \rightarrow \pm \infty} \dot{M}(t)=0$ and $\lim _{t \rightarrow \pm \infty} M(t)=M_{ \pm}$exist. By (6.13),

$$
\sigma\left(M_{ \pm}\right) \cap i \mathbf{R}=\emptyset \text {. }
$$

Let now $\theta \in C^{1}(\mathbf{R}, \mathbf{R})$ satisfy $\operatorname{Supp} \theta \subset J_{t_{m}}, \theta \geq 0$ and $\theta\left(t_{m}\right)=1$. Then, the functions $\theta x_{\ell}: \mathbf{R} \rightarrow W$ and $\theta x_{\ell}^{*}: \mathbf{R} \rightarrow W^{*}, 1 \leq \ell \leq \ell_{m}$, are well defined and of class $C^{1}$ For $s \in[0,1]$ and $t \in \mathbf{R}$, we set

$$
\widetilde{A}(s, t):=\left(\begin{array}{llll}
A(t) & s \theta(t) x_{1}(t) & \cdots & s \theta(t) x_{\ell_{m}}(t) \\
s \theta(t) x_{1}^{*}(t) & & & \\
\vdots & & M(t) & \\
s \theta(t) x_{\ell_{m}}^{*}(t) & & &
\end{array}\right) .
$$

\footnotetext{
${ }^{5}$ Evidently, $\lambda_{m, \ell}(t)$ need not be an eigenvalue of $A(t)$ for $t$ outside $\bar{J}_{t_{m}}$.
} 
Given $s \in[0,1]$, the path $\widetilde{A}(s, \cdot): \mathbf{R} \rightarrow \mathcal{L}\left(W \times \mathbf{C}^{\ell_{m}}, X \times \mathbf{C}^{\ell_{m}}\right)$ is of class $C^{1}$ and $\lim _{t \rightarrow \infty} \widetilde{A}(s, \cdot)=\widetilde{A}_{+}(s):=A_{+} \times M_{+}, \lim _{t \rightarrow-\infty} \widetilde{A}(s, \cdot)=\widetilde{A}_{-}(s):=A_{-} \times M_{-}$and $\lim _{t \rightarrow \pm \infty} \frac{\partial \tilde{A}}{\partial t}(s, \cdot)=0$. By $(6.17), \sigma\left(\widetilde{A}_{ \pm}(s)\right) \cap i \mathbf{R}=\emptyset$. Two more properties of $\tilde{A}$ are given in the next lemma, in which $\widetilde{\mathcal{O}}$ and $\widetilde{\mathcal{S}}_{k}$ denote the sets $\mathcal{O}$ and $\mathcal{S}_{k}$ in (6.1) and (6.4) when $W$ and $X$ are replaced by $W \times \mathbf{C}^{\ell_{m}}$ and $X \times \mathbf{C}^{\ell_{m}}$, respectively.

Lemma 6.3. (i) If $s \in[0,1], \widetilde{A}(s, \cdot)$ satisfies (6.6) with $W$ and $X$ replaced by $W \times \mathbf{C}^{\ell_{m}}$ and $X \times \mathbf{C}^{\ell_{m}}$, respectively.

(ii) If $s \in(0,1]$ and $\xi \in \mathbf{R}$, then $i \xi \in \sigma(\widetilde{A}(s, t))$ if and only if $t=t_{j}$ for some $1 \leq j \leq m-1$ and $\xi=\xi_{j, \ell}$ for some $1 \leq \ell \leq \ell_{j}$. Furthermore, the mapping $(t, \xi) \in \mathbf{R}^{2} \mapsto \widetilde{A}(s, t)-i \xi I$ intersects all the manifolds $\widetilde{\mathcal{S}}_{k}$ transversely, the spectral flow $\operatorname{sf}(A(s, \cdot))$ is well defined and

$$
s f(A(s, \cdot))=-\sum_{j=1}^{m-1} \sum_{\ell=1}^{\ell_{j}} \operatorname{sgn} \operatorname{Re} \dot{\lambda}_{j, \ell}\left(t_{j}\right) .
$$

Proof. (i) When an operator in Banach spaces has the block decomposition $L=\left(\begin{array}{ll}L_{11} & L_{12} \\ L_{21} & L_{22}\end{array}\right)$ with $L_{22}$ invertible, the invertibility of $L$ is equivalent to the invertibility of $L_{11}-L_{12} L_{22}^{-1} L_{21}$ and the norm of $L^{-1}$ is controlled by $\|\left(L_{11}-\right.$ $\left.L_{12} L_{22}^{-1} L_{21}\right)^{-1}\|,\| L_{22}^{-1}\|,\| L_{12} \|$ and $\left\|L_{21}\right\|$. By (6.18), $\widetilde{A}(s, t)-i \xi I$ has such a block decomposition with an obvious choice for the blocks. In particular, the off-diagonal blocks $L_{12}$ and $L_{21}$ are uniformly bounded and

$$
L_{22}^{-1}=(M(t)-i \xi)^{-1}=\operatorname{diag}\left(\frac{-1}{\overline{\lambda_{m, 1}(t)-i \xi}}, \ldots, \frac{-1}{\overline{\lambda_{m, \ell_{m}}(t)-i \xi}}\right)
$$

so that ||$L_{22}^{-1}||$ is uniformly bounded for $t \in \mathbf{R}$ and $|\xi|$ large enough (recall that the functions $\lambda_{m, \ell}$ are constant outside a finite interval). The operator $L_{11}-L_{12} L_{22}^{-1} L_{21}$ is given by

$$
A(t)-i \xi I+s^{2} \sum_{\ell=1}^{\ell_{m}} \frac{\left\langle\cdot, \theta(t) x_{\ell}^{*}(t)\right\rangle}{\overline{\lambda_{m, \ell}(t)-i \xi}} \theta(t) x_{\ell}(t),
$$

a uniformly small perturbation of $A(t)-i \xi I$ when $t \in \mathbf{R}$ and $|\xi|$ large enough. Thus, the boundedness of $\left\|\left(L_{11}-L_{12} L_{22}^{-1} L_{21}\right)^{-1}\right\|$, i.e., (6.6) for $\widetilde{A}(s, \cdot)$, follows from (6.6).

(ii) Suppose $s \in(0,1]$. We begin by characterizing the pairs $(t, \xi)$ such that $i \xi \in \sigma(\widetilde{A}(s, t))$. By $(6.4)$ for $\widetilde{\mathcal{S}}_{k}$, this is equivalent to $\operatorname{ker}(\widetilde{A}(s, t)-i \xi I) \neq\{0\}$. First, we show that there is no such pair if $t=t_{m}$. Since $\theta\left(t_{m}\right)=1$ and $\lambda_{m, \ell}\left(t_{m}\right)=i \xi_{m, \ell}$, we have that $\left(\widetilde{A}\left(s, t_{m}\right)-i \xi I\right)\left(\begin{array}{l}x \\ \alpha\end{array}\right)=0$ with $\alpha=\left(\alpha_{1}, \ldots, \alpha_{\ell_{m}}\right) \in \mathbf{C}^{\ell_{m}}$ if and only if

$$
\left\{\begin{array}{c}
\left(A\left(t_{m}\right)-i \xi\right) x+s \sum_{\ell=1}^{\ell_{m}} \alpha_{\ell} x_{\ell}\left(t_{m}\right)=0, \\
s\left\langle x, x_{\ell}^{*}\left(t_{m}\right)\right\rangle+\alpha_{\ell} i\left(\xi_{m, \ell}-\xi\right)=0,1 \leq \ell \leq \ell_{m} .
\end{array}\right.
$$

The first relation shows that $\left(A\left(t_{m}\right)-i \xi I\right) x \in Z_{m}$ (see (6.9)). Since $A\left(t_{m}\right)-i \xi I$ maps $Z_{m}$ into $Z_{m}$ and is an isomorphism of $W_{m}$ onto $X_{m}$, as noted earlier, it follows that $x \in Z_{m}$, whence $x=\sum_{\ell=1}^{\ell_{m}} \beta_{\ell} x_{\ell}\left(t_{m}\right)$ with $\beta_{\ell} \in \mathbf{C}$. By substituting into the second relation in (6.21) and by using (6.14), we find $s \beta_{\ell}+\alpha_{\ell} i\left(\xi_{m, \ell}-\xi\right)=$ $0,1 \leq j \leq \ell_{m}$ and hence $\beta_{\ell}=-\frac{\alpha_{\ell} i\left(\xi_{m, \ell}-\xi\right)}{s}, 1 \leq j \leq \ell_{m}$. Now, returning to the 
first relation in (6.21), we find $\left(\frac{\left(\xi_{m, \ell}-\xi\right)^{2}}{s}+s\right) \alpha_{\ell}=0$, whence $\alpha_{\ell}=0$ since $s>0$. Thus, $\alpha=0$ and $x=0$. This shows that $\sigma\left(\widetilde{A}\left(s, t_{m}\right)\right) \cap i \mathbf{R}=\emptyset$.

From now on, we assume $t \neq t_{m}$. We shall prove below that $i \xi \notin \sigma(\widetilde{A}(s, t))$ whenever $t \in \bar{J}_{t_{m}}$, so that $i \xi \in \sigma(\widetilde{A}(s, t))$ only if $t \in \mathbf{R} \backslash \bar{J}_{t_{m}}$, in which case $\widetilde{A}(s, t)=A(t) \times M(t)$. (This crucial step was omitted in the proof of [34, Theorem 4.25].) When $t \in \mathbf{R} \backslash \bar{J}_{t_{m}}, M(t)$ has no imaginary eigenvalue by (6.13), so that the imaginary eigenvalues of $A(t) \times M(t)$ coincide with the imaginary eigenvalues of $A(t)$. By (6.15), these eigenvalues are obtained for $(t, \xi)=\left(t_{j}, \xi_{j, \ell}\right)$ for some $1 \leq j \leq m-1$ and $1 \leq \ell \leq \ell_{j}$. Furthermore, since $\operatorname{Supp} \theta \subset J_{t_{m}}, \widetilde{A}(s, t)$ coincides with $A(t) \times M(t)$ for all $t$ in an open neighborhood of $t_{j}$ and then the transversality of the intersection of $\widetilde{A}(s, t)-i \xi I$ with $\widetilde{\mathcal{S}}_{k}$ at $\left(t_{j}, \xi_{j, \ell}\right)$ follows at once from the transversality of the intersection of $A(t)-i \xi I$ with $\mathcal{S}_{k}$ at $\left(t_{j}, \xi_{j, \ell}\right)$. Also, for $t$ near $t_{j}, 1 \leq j \leq m-1$, the eigenvalues of $A(t) \times M(t)$ and $A(t)$ near $\xi_{j, \ell}$ are the same and hence given by the functions $\lambda_{j, \ell}(t)$. Thus, from part (i) and the comments preceding the lemma, the spectral flow $s f(A(s, \cdot))$ is well defined and given by (6.19).

Suppose then that $t \in \bar{J}_{t_{m}} \backslash\left\{t_{m}\right\}$. It follows from (6.13) that $M(t)-i \xi$ is invertible for all $\xi \in \mathbf{R}$, so that $i \xi \notin \sigma(\widetilde{A}(s, t))$ if and only if the operator (6.20) is invertible. Since this operator is a finite dimensional perturbation of $A(t)-i \xi$, its invertibility is equivalent to the triviality of its null space (recall that $A(t)$ has compact resolvent).

Let $x \in W$ be such that

$$
(A(t)-i \xi I) x+s^{2} \sum_{\ell=1}^{\ell_{m}} \frac{\left\langle x, \theta(t) x_{\ell}^{*}(t)\right\rangle}{\lambda_{m, \ell}(t)-i \xi} \theta(t) x_{\ell}(t)=0,
$$

so that $(A(t)-i \xi I) x \in Z_{m}(t)$ (see (6.11)). By (6.12), we can write $x=z+y$ with $z=\sum_{\ell=1}^{\ell_{m}} \alpha_{\ell} x_{\ell}(t) \in Z_{m}(t)$ and $y \in W_{m}$ and, recalling (6.14), (6.22) becomes

$$
\sum_{\ell=1}^{\ell_{m}}\left(\lambda_{m, \ell}(t)-i \xi+\frac{s^{2} \theta^{2}(t)}{\lambda_{m, \ell}(t)-i \xi}\right) \alpha_{\ell} x_{\ell}(t)+(A(t)-i \xi I) y=0 .
$$

From the second part of (6.11), we infer that $(A(t)-i \xi I) y=0$ and hence that $\left(\lambda_{m, \ell}(t)-i \xi+\frac{s^{2} \theta^{2}(t)}{\lambda_{m, \ell}(t)-i \xi}\right) \alpha_{\ell}=0$ for $1 \leq \ell \leq \ell_{m}$. Since $t \in \bar{J}_{t_{m}} \backslash\left\{t_{m}\right\}$, it follows from (6.15) that $t \notin\left\{t_{1}, \ldots, t_{m}\right\}$ and hence that $A(t)-i \xi I$ is one-to-one. Thus, $y=0$. Also, $\lambda_{m, \ell}(t)-i \xi+\frac{s^{2} \theta^{2}(t)}{\lambda_{m, \ell}(t)-i \xi}=\frac{\left|\lambda_{m, \ell}(t)-i \xi\right|^{2}+s^{2} \theta^{2}(t)}{\overline{\lambda_{m, \ell}(t)-i \xi}} \neq 0$ by (6.13), whence $\alpha_{\ell}=0$ for $1 \leq \ell \leq \ell_{m}$. This shows that $x=0$ and the proof is complete.

Lemma 6.4. Suppose that, in addition, $X$ is a Banach space with UMD and that A satisfies the conditions (H1) to (H5). Then,

(i) $X \times \mathbf{C}^{\ell_{m}}$ is a Banach space with $U M D$,

(ii) for $s \in[0,1], \widetilde{A}(s, \cdot)$ satisfies (H1) to (H5) with $X$ and $W$ replaced by $X \times \mathbf{C}^{\ell_{m}}$ and $W \times \mathbf{C}^{\ell_{m}}$, respectively.

Proof. (i) This follows from $\operatorname{dim} \mathbf{C}^{\ell_{m}}<\infty$ and the fact that the product of two Banach spaces with UMD has the UMD property (see the Introduction).

(ii) The conditions $(\mathrm{H} 1),(\mathrm{H} 2),(\mathrm{H} 3)$ and $(\mathrm{H} 5)$ for $\widetilde{A}(s, \cdot)$ are trivial and were already noticed earlier. It remains to prove (1.3) for $\widetilde{A}(s, t)$, assuming the same 
for $A(t)$. First, condition ( 6.6) holds trivially with $A(t)$ replaced by $M(t)$ (and $X$ and $W$ replaced by $\mathbf{C}^{\ell_{m}}$ ) and implies (2.5) for $A_{0}=M(t)$ since finite dimensional spaces are Hilbert spaces (so that boundedness and r-boundedness coincide). Thus, (2.5) holds for both $A(t)$ and $M(t)$, the former by (1.3) and Theorem 2.5. As a result, (2.5) holds for $A_{0}=A(t) \times M(t)$ since the product of two r-bounded sets is itself r-bounded (see e.g. [32]). By using once again Theorem 2.5, it follows that $\widetilde{A}(0, t)=A(t) \times M(t)$ satisfies (1.3). Next, $\widetilde{A}(s, t)$ is a finite dimensional perturbation of $\widetilde{A}(0, t)$. Thus, $\widetilde{A}(s, t)$ is an $\widetilde{A}(0, t)$-bounded perturbation of $\widetilde{A}(0, t)$ with $\widetilde{A}(0, t)$-bound 0 . That $\widetilde{A}(s, t)$ satisfies $(1.3)$ thus follows from Theorem 2.3.

We are finally in a position to prove the desired result that index $D_{A}=s f(A)$. Recall that $\mathcal{O}$ and $\mathcal{S}_{k}$ are defined in (6.1) and (6.2), respectively, and the spaces $\mathcal{W}^{p}$ to $\mathcal{X}^{p}$ are defined in (3.1).

Theorem 6.5. Let $X$ be a Banach space with UMD and let $(A(t))_{t \in \mathbf{R}}$ be a family of unbounded operators on $X$ satisfying the assumptions (H1) to (H5). Suppose also that $A: \mathbf{R} \rightarrow \mathcal{L}(W, X)$ is $C^{1}$, that $\lim _{t \rightarrow \pm \infty} \dot{A}(t)=0$ and that the mapping $(t, \xi) \in \mathbf{R}^{2} \mapsto A(t)-i \xi I \in \mathcal{O}$ intersects each manifold $\mathcal{S}_{k}$ transversely. Then, the operator $D_{A}$ is Fredholm from $\mathcal{W}^{p}$ to $\mathcal{X}^{p}$ for every $p \in(1, \infty)$, the spectral flow $s f(A)$ is well defined and index $D_{A}=s f(A)$.

Proof. That $D_{A}$ is Fredholm is Theorem 4.2 and that $s f(A)$ is defined follows from our assumptions about $A$. If $\sigma(A(t)) \cap i \mathbf{R}=\emptyset$ for all $t \in \mathbf{R}$, then $s f(A)=0$ while index $D_{A}=0$ by Theorem 5.2 , so that index $D_{A}=s f(A)$. Next, consistent with Lemma 6.2 (ii), assume that $\sigma(A(t)) \cap i \mathbf{R} \neq \emptyset$ for exactly one value $t=t_{1}$, so that $m=1$ in Lemma 6.2. With the notation of that lemma, we have

$$
s f(A)=-\sum_{\ell=1}^{\ell_{1}} \operatorname{sgn} \operatorname{Re} \dot{\lambda}_{1, \ell}\left(t_{1}\right) .
$$

Let $\widetilde{A}(s, t)$ be given by $(6.18)$ with $m=1$. By Lemma 6.4 and Theorem 4.2 , the operator $D_{\tilde{A}(s, \cdot)}$ is Fredholm from $\mathcal{W}^{p}$ to $\mathcal{X}^{p}$ for all $s \in[0,1]$. By the local constancy of the index, it follows that $i n \operatorname{dex} D_{\tilde{A}(0, \cdot)}=i n d e x D_{\tilde{A}(1, \cdot)}$. Now, by Lemma 6.3 (ii), we have $\sigma(\widetilde{A}(1, \cdot)) \cap i \mathbf{R}=\emptyset$ for all $t \in \mathbf{R}$, so that $i n \operatorname{dex} D_{\tilde{A}(1, \cdot)}=0$ by Theorem 5.2. Thus, index $D_{\tilde{A}(0, \cdot)}=0$. But $\widetilde{A}(0, t)=A(t) \times M(t)$, so that index $D_{\tilde{A}(0, \cdot)}=\operatorname{index} D_{A}+\operatorname{index} D_{M}$ as a straightforward verification reveals, so that

$$
\text { index } D_{A}=-\operatorname{index} D_{M}
$$

Since $M$ is a finite dimensional path, the relation index $D_{M}=s f(M)$ holds (Remark 6.2). Now, it is trivial from (6.16) with $m=1$ that $M$ satisfies the conditions required for $s f(M)$ to be given by (6.8) with $m=1$ and $\lambda_{1, \ell}(t)$ replaced by $-\bar{\lambda}_{1, \ell}(t)$. As a result,

$$
\text { index } D_{M}=\sum_{\ell=1}^{\ell_{1}} \operatorname{sgn} \operatorname{Re} \dot{\lambda}_{1, \ell}\left(t_{1}\right) \text {. }
$$

The relation $i$ dex $D_{A}=s f(A)$ thus follows from (6.23), (6.24) and (6.25).

To complete the proof, we consider general case and use the notation of Lemma 6.2 (iii). From the above, we may assume $m>1$ and, by induction, that the 
relation $i n d e x D_{A}=s f(A)$ is true whenever $\sigma(A(t))$ intersects $i \mathbf{R}$ at $m-1$ distinct points. Arguing as above, we obtain index $D_{\tilde{A}(0, \cdot)}=\operatorname{index} D_{\tilde{A}(1, \cdot)}=\operatorname{sf}(A(s, \cdot))=$ $-\sum_{j=1}^{m-1} \sum_{\ell=1}^{\ell_{j}} \operatorname{sgn} \operatorname{Re} \dot{\lambda}_{j, \ell}\left(t_{j}\right)$ by the hypothesis of induction and Lemma 6.3 (ii). Next, $\widetilde{A}(0, t)=A(t) \times M(t)$, so that, as before, index $D_{\tilde{A}(0, \cdot)}=$ index $D_{A}+$ index $D_{M}$ and index $D_{M}=s f(M)$ by Remark 6.2, while $s f(M)=\sum_{\ell=1}^{\ell_{1}} \operatorname{sgn} \operatorname{Re} \dot{\lambda}_{m, \ell}\left(t_{1}\right)$. Thus, index $D_{A}=-\sum_{j=1}^{m} \sum_{\ell=1}^{\ell_{j}} \operatorname{sgn} \operatorname{Re} \dot{\lambda}_{j, \ell}\left(t_{j}\right)=s f(A)$ by $(6.8)$.

The characterization of index $D_{A}$ in Theorem 6.5 along with the approximation result of Lemma 6.6 below is very useful to calculate index $D_{A}$ in cases more complicated than the ones considered in the previous section. An example is given Theorem 6.7.

Lemma 6.6. Let $(A(t))_{t \in \mathbf{R}}$ be a family of unbounded operators on $X$ satisfying ${ }^{6}$ the assumptions (H1) to (H5). Given $\varepsilon>0$, there is a $C^{1}$ path $A_{\varepsilon}: \mathbf{R} \rightarrow \mathcal{L}(W, X)$ with the following properties:

(i) $\lim _{t \rightarrow \pm \infty} A_{\varepsilon}(t)=A_{ \pm}, \lim _{t \rightarrow \pm \infty} \dot{A}(t)=0$,

(ii) $\sup _{t \in \mathbf{R}}\left\|A(t)-A_{\varepsilon}(t)\right\|_{\mathcal{L}(W, X)} \leq \varepsilon$,

(iii) the mapping $(t, \xi) \in \mathbf{R}^{2} \mapsto A_{\varepsilon}(t)-i \xi I \in \mathcal{O}$ intersects each manifold $\mathcal{S}_{k}$ transversely,

(iv) $\left(A_{\varepsilon}(t)\right)_{t \in \mathbf{R}}$ satisfies the assumptions (H1) to (H5).

In particular, if $X$ has the UMD property and $\varepsilon>0$ is small enough, then index $D_{A}=$ index $D_{A_{\varepsilon}}=\operatorname{sf}\left(A_{\varepsilon}\right)$ when $D_{A}$ is viewed as an operator from $\mathcal{W}^{p}$ to $\mathcal{X}^{p}, p \in(1, \infty)$.

Proof. It is straightforward to check that a $C^{1}$ path $A_{\varepsilon}$ can be found that satisfies (i) and (ii). By Lemma 6.2 (ii), $A_{\varepsilon}$ can be modified so as to satisfy (iii) as well. Then, after shrinking $\varepsilon>0$ if necessary, it follows from Corollary 2.4 that $A_{\varepsilon}$ satisfies (iv). The "in particular" part follows at once from the local constancy of index $D_{A}$ and Theorem 6.5.

THEOREM 6.7. Let $(A(t))_{t \in \mathbf{R}}$ be a family of unbounded operators on $X$ satisfying the assumptions (H1) to (H5). Suppose also that $A_{-}$has only a finite number of eigenvalues with positive real part, so that the sum of the corresponding generalized eigenspaces has finite dimension ${ }^{7} \nu_{-}$. Then,

(i) $A_{+}$has a finite number of eigenvalues with positive real part and the sum of the corresponding generalized eigenspaces has finite dimension $\nu_{+}$.

(ii) If $X$ has the UMD property, then index $D_{A}=\nu_{-}-\nu_{+}$when $D_{A}$ is viewed as an operator from $\mathcal{W}^{p}$ to $\mathcal{X}^{p}, p \in(1, \infty)$.

Proof. By Lemma 6.6 and the local constancy of the index of $D_{A}$, we may assume with no loss of generality that $A$ is a $C^{1}$ path such that $\lim _{t \rightarrow \pm \infty} A_{\varepsilon}(t)=$ $A_{ \pm}, \lim _{t \rightarrow \pm \infty} \dot{A}(t)=0$ and the mapping $(t, \xi) \in \mathbf{R}^{2} \mapsto A(t)-i \xi I \in \mathcal{O}$ intersects each manifold $\mathcal{S}_{k}$ transversely. Both (i) and (ii) are trivial if $\sigma(A(t)) \cap i \mathbf{R}=\emptyset$ for all $t \in \mathbf{R}$, so that we may assume that $m \geq 1$ in Lemma 6.2 (iii). Evidently, we may also assume that $t_{1}<\cdots<t_{m}$ in that lemma. Denote by $\nu(t)$ the dimension (finite or infinite) of the sum of the generalized eigenspace associated with the eigenvalues of $A(t)$ with positive real part. Since $A(t)$ has compact resolvent and $\lim _{t \rightarrow-\infty} A(t)=A_{-}$, it follows from standard perturbation theory of isolated

\footnotetext{
${ }^{6}$ Here, there is no need to assume that $X$ has the UMD property.

${ }^{7}$ Because $A_{-}$has compact resolvent.
} 
eigenvalues (Kato [25]) that $\nu(t)=\nu_{-}$as $t \rightarrow-\infty$. In fact, similar arguments easily show that $\nu(t)=\nu_{-}$for $t \in\left(-\infty, t_{1}\right)$.

As $t$ crosses $t_{1}$, the set of eigenvalues of $A(t)$ with positive real part loses (exactly) the algebraically simple eigenvalues $\lambda_{1, \ell}(t)$ with $\dot{\lambda}_{1, \ell}\left(t_{1}\right)<0$ and gains (exactly) those with $\dot{\lambda}_{1, \ell}\left(t_{1}\right)>0$. The justification of this statement requires using condition (1.4) for $t=t_{1}$ (in the equivalent form given in Theorem 2.5) showing that if $\lambda^{(k)}$ is a sequence of eigenvalues of $A\left(t^{(k)}\right)$ approaching the imaginary axis as $t^{(k)} \rightarrow$ $t_{1}$, then $\lambda^{(k)}$ must tend to the set $\left\{i \xi_{1,1}, \ldots, i \xi_{1, \ell_{1}}\right\}$ (hence cannot escape to infinity). Thus, as $t$ crosses $t_{1}, \nu(t)$ passes from $\nu_{-}$to $\nu_{-}+\sum_{\ell=1}^{\ell_{1}} \dot{\lambda}_{1, \ell}\left(t_{1}\right)$. By the same arguments, $\nu(t)$ remains constant in $\left(t_{1}, t_{2}\right), \ldots,\left(t_{m}, \infty\right)$ and increases by $\sum_{\ell=1}^{\ell_{j}} \dot{\lambda}_{j, \ell}\left(t_{j}\right)$ when $t$ crosses $t_{j}$. This shows that $\nu_{+}=\nu_{-}+\sum_{j=1}^{m} \sum_{\ell=1}^{\ell_{j}} \operatorname{sgn} \operatorname{Re} \dot{\lambda}_{j, \ell}\left(t_{j}\right)=$ $\nu_{-}-s f(A)$, so that $\nu_{+}<\infty$ and $\nu_{-}-\nu_{+}=s f(A)=\operatorname{index} D_{A}$.

There is an obvious variant of Theorem 6.7 if $A_{+}$rather than $A_{-}$is assumed to have only a finite number of eigenvalues with positive real part. An equally obvious variant is given by the case when $A_{-}$or $A_{+}$has only a finite number of eigenvalues with negative real part.

REMARK 6.3. It should not be inferred from Theorem 6.7 that index $D_{A}$ always depends only upon the endpoints $A_{ \pm}$. This was proved only when $A_{ \pm}$have finitely many eigenvalues with positive or negative real parts.

\section{The case of differential operators}

We complement the previous sections with some comments regarding the verification of the assumptions (H1) to (H5) when $(A(t))_{t \in \mathbf{R}}$ is a family of second order elliptic differential operators on a bounded open subset $\Omega \subset \mathbf{R}^{N}$ with sufficiently smooth boundary $\partial \Omega$. We choose $X=L^{q}(\Omega)$ for some $q \in(1, \infty)$ and let $W$ denote a closed subspace of $W^{2, q}(\Omega)$ incorporating boundary conditions such that $\mathcal{D}(\Omega) \subset W$.

As pointed out in the Introduction, $X=L^{q}(\Omega)$ has the UMD property. In the setting just outlined above, (H1) holds and the same thing is true of (H2) and (H3) under self-evident simple conditions about the coefficients of $A(t)$. Since $\sigma\left(A_{ \pm}\right)$is discrete when (H1) to (H4) hold (Theorem 2.1 (ii)), (H5) is not a severe additional restriction. The verification of (H4) is more delicate. Since (H4) is just condition (2.3) of Theorem 2.3 with $A_{0}=A(t)$ and $t \in \overline{\mathbf{R}}$, it will be clearer to discuss the verification of (2.3), first in the abstract setting and next when, as above, $A_{0}$ is a second order elliptic differential operator.

Assume that $\left\|\lambda R\left(\lambda, A_{0}\right)\right\|_{\mathcal{L}(X)}$ is bounded in the complement of a sector $\Sigma_{\sigma_{0}}$ with angle $\sigma_{0}<\frac{\pi}{2}$ around the negative real axis (so that $A_{0}$ generates a bounded holomorphic semigroup) and that $A_{0}$ has a bounded $H^{\infty}\left(\Sigma_{\sigma_{0}}\right)$ functional calculus. As shown by Kalton and Weis ${ }^{8}$ [24, Theorem 5.3, part 3], if $\nu>\sigma_{0}$ the set $\left\{2^{n} s R\left(2^{n} s e^{ \pm i \nu}, A_{0}\right): n \in \mathbf{Z}\right\}$ is r-bounded with r-bound independent of $s>0$. (In [24], $A_{0}$ is $-A_{0}$ so that $\Sigma_{\sigma_{0}}$ is a sector around the positive real axis.) Thus, (2.3) follows by choosing $\nu=\frac{\pi}{2}$, which is possible since $\sigma_{0}<\frac{\pi}{2}$, and $s=|a|$. More generally, from the above and Theorem 2.3, (2.3) continues to hold if $A_{0}-\omega_{0} I$ has a bounded $H^{\infty}\left(\Sigma_{\sigma_{0}}\right)$ functional calculus for some $\omega_{0}>0$ and $\sigma_{0}<\frac{\pi}{2}$.

\footnotetext{
${ }^{8}$ In the terminology of $[\mathbf{2 4}]$, this requires "property $\Delta$ ", which holds in all spaces with UMD, and even in the more general class of spaces with "analytic UMD" ([24, Proposition 3.2]).
} 
The above changes the problem into finding conditions ensuring that $A_{0}$ or, more generally, $A_{0}-\omega_{0} I$, has a bounded $H^{\infty}\left(\Sigma_{\sigma_{0}}\right)$ functional calculus, an issue investigated by Duong and Robinson [19] for abstract operators $A_{0}$ on $X=L^{q}(\Omega)$. They show that the question can be reduced to the existence of suitable bounds for the kernel of the semigroup generated by $A_{0}$. The specialization of these ideas to the case of a second order elliptic differential operator $A_{0}$ was subsequently considered by Arendt and ter Elst [7] (see also Duong and McIntosh [18]). The net result is that $A_{0}-\omega_{0} I$ has a bounded $H^{\infty}\left(\Sigma_{\sigma_{0}}\right)$ functional calculus for some $\omega_{0}>0$ and $\sigma_{0}<\frac{\pi}{2}$ under familiar boundedness and regularity assumptions about the coefficients of $A_{0}$ and various boundary conditions on $\partial \Omega$. See [7, Theorems 3.1 and 4.4]; that the desired functional calculus properties hold follows from [7, Theorems 5.4 and 5.7].

In summary, just like the other hypotheses, the assumption (H4) is quite reasonable when $A(t)$ is a second order elliptic operator for $t \in \overline{\mathbf{R}}$. While the justification given here is rather convoluted, a more direct argument, if any, remains to be found. In that regard, it may be helpful to observe that since $X=L^{q}(\Omega)$, the simpler formulation of the r-boundedness condition in Remark 1.1 is available.

REMARK 7.1. Theorem 5.3 is relevant in the above setting since, due to the compactness of the embedding $W^{1, q}(\Omega) \hookrightarrow L^{q}(\Omega)$, the operator $C(t)$ may represent a lower order perturbation of $A(t)$. This is to say that, if convenient, the verification of (H1) to (H5) for $A(t)$ may be done after modifying the lower order terms.

REMARK 7.2. Little seems to be available for higher order elliptic boundary value problems, but elliptic systems on $\mathbf{R}^{N}$ and on compact manifolds also have a bounded $H^{\infty}$ functional calculus; see Amann, Hieber and Simonett [6]. However, when $X=L^{q}\left(\mathbf{R}^{N}\right)$ and $W=W^{2, q}\left(\mathbf{R}^{N}\right)$, the embedding $W \hookrightarrow X$ is not compact and (H1) fails to hold.

A trivial but important remark is that the assumptions (H1) to (H5) are unaffected by changing $A$ into $-A$. As a result, if $\left(A_{1}(t)\right)_{t \in \mathbf{R}} \subset \mathcal{L}\left(W_{1}, X_{1}\right)$ and $\left(A_{2}(t)\right)_{t \in \mathbf{R}} \subset \mathcal{L}\left(W_{2}, X_{2}\right)$ satisfy (H1) to (H5) then also $(A(t))_{t \in \mathbf{R}} \subset \mathcal{L}\left(W_{1} \times\right.$ $\left.W_{2}, X_{1} \times X_{2}\right)$ defined by $A(t):=A_{1}(t) \times\left(-A_{2}(t)\right)=\left(\begin{array}{cc}A_{1}(t) & 0 \\ 0 & -A_{2}(t)\end{array}\right)$, satisfies (H1) to (H5) with $W=D(A(t))=W_{1} \times W_{2}$. In general, neither $A(t)$ nor $-A(t)$ generates a semigroup and hence the previous arguments cannot be used directly to justify the validity of (H4), but this follows at once from the elementary fact that the product of two r-bounded subsets is r-bounded (see for instance [32]). Since $X_{1} \times X_{2}$ has the UMD property along with $X_{1}$ and $X_{2}$, the index theorems of this paper are available with the operator $D_{A}=\frac{d}{d t}-\left(\begin{array}{cc}A_{1} & 0 \\ 0 & -A_{2}\end{array}\right)$ and hence with the Hamiltonian-like operator

$$
J D_{A}=J \frac{d}{d t}-\left(\begin{array}{cc}
0 & A_{2} \\
A_{1} & 0
\end{array}\right)
$$

where $J=\left(\begin{array}{ll}0 & -I \\ I & 0\end{array}\right)$. More generally, Theorem 5.3 shows that the index of $D_{A}$ above is unaffected by suitable lower order perturbations. 


\section{Initial value problems on the half-line and $L^{p}$ maximal regularity}

Given $p \in(1, \infty)$ and a family $A:=(A(t))_{t \in \overline{\mathbf{R}}_{+}}$of generators of semigroups on a Banach space $X$, where $\mathbf{R}_{+}:=(0, \infty)$, the so-called $L^{p}$ maximal regularity question for the Cauchy problem may be phrased as follows: Find conditions about $A$ ensuring the existence of a constant $C>0$ such that

$$
\|u\|_{W^{1, p}\left(\mathbf{R}_{+}, X\right)}+\|A u\|_{L^{p}\left(\mathbf{R}_{+}, X\right)} \leq C\|f\|_{L^{p}\left(\mathbf{R}_{+}, X\right)},
$$

for all $f \in L^{p}\left(\mathbf{R}_{+}, X\right)$ and corresponding solutions $u$ of the Cauchy problem

$$
\left\{\begin{array}{l}
\frac{d u}{d t}=A u+f \text { in } \mathbf{R}_{+} \\
u(0)=0 .
\end{array}\right.
$$

A frequently discussed variant and indeed a simpler problem, arises when $\mathbf{R}_{+}$is replaced by a finite interval $(0, T)$.

There is by now a fairly large literature about $L^{p}$ maximal regularity, especially when $A(t)=A_{0}$ is constant. If $X$ is a Hilbert space and $A_{0}$ generates a holomorphic semigroup on $X$, the issue was resolved by de Simon [36] in 1964, but in spite of various subsequent results in special cases, a broader treatment remained elusive until Dore and Venni [17] found a generalization in Banach spaces with UMD. In such spaces, a necessary and sufficient condition for $L^{p}$ maximal regularity in problems with constant coefficients was finally obtained by Weis $[\mathbf{3 8}]$ as an application of his operator-valued generalization of Mikhlin's multiplier theorem.

Most treatments of the variable coefficients case introduce Hölder type assumptions about the coefficients via commutator conditions and are limited to finite intervals. The domains may or may not depend upon $t$. See for instance Acquistapace and Terreni [2], Giga, Giga and Sohr [21], Yamamoto [39], Monniaux and Prüss [29], Hieber and Monniaux [23], Prüss and Schnaubelt [31], among others. In some of these works, a special structure of $X$, for example Hilbert or $L^{q}$ space, is assumed. In [31], the $L^{p}$ maximal regularity problem is discussed assuming only the continuity of the coefficients, but only on finite intervals.

In this section, we establish the $L^{p}$ maximal regularity on the half-line for problems with continuous coefficients as an application of the earlier results. This approach shows that, in general, the inequality (8.1) can only hold when $f$ satisfies a finite number of "compatibility" condition obtained via the null-space of $D_{-A^{*}}$ on the whole line, which can hardly be discovered by confining the attention to the interval $\mathbf{R}_{+}$.

We begin with a local uniqueness result.

LEMma 8.1. Let $X$ be a Banach space with $U M D$ and let $(A(t))_{t \in \mathbf{R}}$ be a family of unbounded operators on $X$ satisfying the assumptions (H1) to (H5).

(i) If $A(0)$ generates a holomorphic semigroup, there is $\varepsilon>0$ such that the only solution $u \in W^{1, p}((0, \varepsilon), X) \cap L^{p}((0, \varepsilon), W)$ of

$$
\left\{\begin{array}{l}
\frac{d u}{d t}=A u \text { in }(0, \varepsilon) \\
u(0)=0
\end{array}\right.
$$

is $u=0$.

(ii) If $A(t)$ generates a holomorphic semigroup for all $t \geq 0$, then the only solution $u \in W^{1, p}\left(\mathbf{R}_{+}, X\right) \cap L^{p}\left(\mathbf{R}_{+}, W\right)$ of

$$
\left\{\begin{array}{l}
\frac{d u}{d t}=A u \text { in } \mathbf{R}_{+} \\
u(0)=0
\end{array}\right.
$$


is $u=0$.

Proof. (i) By (H4) with $t=0$ and the assumption that $A(0)$ generates a holomorphic semigroup, the operator $\frac{d u}{d t}-A(0)$ has maximal $L^{p}$ regularity on finite intervals (see [38, Remark 4.7] or [32]) and hence there is a constant $C_{T}>0$ such that

$$
\|u\|_{W^{1, p}((0, T), X)}+\|A(0) u\|_{L^{p}((0, T), X)} \leq C_{T}\|f\|_{L^{p}((0, T), X)},
$$

for all $f \in L^{p}((0, T), X)$ and corresponding (unique) solution $u \in W^{1, p}((0, T), X) \cap$ $L^{p}((0, T), W)$ of the Cauchy problem

$$
\left\{\begin{array}{l}
\frac{d u}{d t}=A(0) u+f \text { in }(0, T), \\
u(0)=0 .
\end{array}\right.
$$

The uniqueness property with $T=\varepsilon$ implies that if $0<\varepsilon<1$ and $f \in$ $L^{p}((0,1), X)$, then the solution $u$ of $(8.6)$ on $(0, \varepsilon)$ is simply the restriction to $(0, \varepsilon)$ of the solution on $(0,1)$ and then

$$
\|u\|_{W^{1, p}((0, \varepsilon), X)}+\|A(0) u\|_{L^{p}((0, \varepsilon), X)} \leq C_{1}\|f\|_{L^{p}((0,1), X)},
$$

where $C_{1}>0$ is the constant corresponding to $T=1$ in (8.5). Now, if $f \in$ $L^{p}((0, \varepsilon), X)$, extend $f$ by 0 outside $(0, \varepsilon)$, so that $\|f\|_{L^{p}((0, \varepsilon), X)}=\|f\|_{L^{p}((0,1), X)}$ and, by $(8.7)$

$$
\|u\|_{W^{1, p}((0, \varepsilon), X)}+\|A(0) u\|_{L^{p}((0, \varepsilon), X)} \leq C_{1}\|f\|_{L^{p}((0, \varepsilon), X)},
$$

with no modification of $C_{1}$.

Let then $u \in W^{1, p}((0, \varepsilon), X) \cap L^{p}((0, \varepsilon), W)$ solve (8.3), so that $u$ solves (8.6) with $T=\varepsilon$ and $f=(A-A(0)) u$. By (8.8),

$$
\begin{array}{r}
\|u\|_{W^{1, p}((0, \varepsilon), X)}+\|A(0) u\|_{L^{p}((0, \varepsilon), X)} \leq C_{1}\|(A-A(0)) u\|_{L^{p}((0, \varepsilon), X)} \leq \\
C_{1} \sup _{t \in[0, \varepsilon]}\|A(t)-A(0)\|_{\mathcal{L}(W, X)}\|u\|_{L^{p}((0, \varepsilon), W) .}
\end{array}
$$

Since the norm of $W$ is (equivalent to) the graph norm of $A(0)$, we have

$$
\begin{aligned}
\|u\|_{L^{p}((0, \varepsilon), W) \leq} \leq & 2^{\frac{p-1}{p}}\left(\|u\|_{L^{P}((0, \varepsilon), X)}+\|A(0) u\|_{L^{p}((0, \varepsilon), X)}\right) \leq \\
& 2^{\frac{p-1}{p}}\left(\|u\|_{W^{1, p}((0, \varepsilon), X)}+\|A(0) u\|_{L^{p}((0, \varepsilon), X)}\right)
\end{aligned}
$$

and hence, by (8.9),

$$
2^{\frac{p}{p-1}}\|u\|_{L^{p}((0, \varepsilon), W)} \leq C_{1} \sup _{t \in[0, \varepsilon]}\|A(t)-A(0)\|\left\|_{\mathcal{L}(W, X)}\right\| u \|_{L^{p}((0, \varepsilon), W)} .
$$

By (H2), $C_{1} \sup _{t \in[0, \varepsilon]}\|A(t)-A(0)\|_{\mathcal{L}(W, X)}<2^{\frac{p}{p-1}}$ if $\varepsilon>0$ is small enough and then $u=0$.

(ii) By contradiction, suppose that $u \in W^{1, p}\left(\mathbf{R}_{+}, X\right) \cap L^{p}\left(\mathbf{R}_{+}, W\right)$ is a nonzero solution of (8.4). It follows from part (i) that $u=0$ on some subinterval $\left(0, t_{0}\right)$, so that and we may assume that $\left(0, t_{0}\right)$ is the largest subinterval of $\mathbf{R}_{+}$in which $u=0$. Note that $t_{0}<\infty$ since $u \neq 0$ and that $u\left(t_{0}\right)=0$ by continuity.

Given $\varepsilon>0$, we have $u \in W^{1, p}\left(\left(t_{0}, t_{0}+\varepsilon\right), X\right) \cap L^{p}\left(\left(t_{0}, t_{0}+\varepsilon\right), W\right)$ and

$$
\left\{\begin{array}{l}
\frac{d u}{d t}=A u \text { in }\left(t_{0}, t_{0}+\varepsilon\right) \\
u\left(t_{0}\right)=0
\end{array}\right.
$$

and since $A\left(t_{0}\right)$ generates a holomorphic semigroup, it follows from part (i) with $A(t)$ replaced by $A\left(t+t_{0}\right)$ (which does not affect (H1) to (H5)) that $u=0$ in 
$\left(t_{0}, t_{0}+\varepsilon\right)$ if $\varepsilon>0$ is small enough. A contradiction is then reached with the maximality of $\left(0, t_{0}\right)$.

REMARK 8.1. Instead of (ii) in Lemma 8.1, assume that $-A(t)$ generates a holomorphic semigroup for all $t \leq 0$. Then, the only solution $u \in W^{1, p}\left(\mathbf{R}_{-}, X\right) \cap$ $L^{p}\left(\mathbf{R}_{-}, W\right)$ of

$$
\left\{\begin{array}{l}
\frac{d u}{d t}=A u \text { in } \mathbf{R}_{-}, \\
u(0)=0,
\end{array}\right.
$$

is $u=0$. This follows from Lemma 8.1 after setting $u(t)=v(-t)$.

Lemma 8.2 below is the bridge between problems on the whole line and problems on the half line.

LEMMA 8.2. Let $X$ be a Banach space with $U M D$ and let $(A(t))_{t \in \mathbf{R}}$ be a family of unbounded operators on $X$ satisfying not only the assumptions (H1) to (H5) but also

(i) $A(t)=A(0)$ for every $t \leq 0$.

(ii) $A(0)\left(=A_{-}\right)$generates a holomorphic semigroup on $X$.

Denote by $X_{0}^{+}$the direct sum of the generalized eigenspaces of $A(0)$ associated with eigenvalues with positive real part. Then, $\operatorname{dim} X_{0}^{+}<\infty$ and, given $p \in(1, \infty)$, the following property holds: If $u \in \mathcal{W}^{p}$ and $D_{A} u=0$ a.e. on $\mathbf{R}_{-}$, then $u(t) \in X_{0}^{+}$for every $t \in \overline{\mathbf{R}}_{-}$. In particular, if $A(0)$ generates a bounded holomorphic semigroup on $X$, then $u=0$ on $\overline{\mathbf{R}}_{-}$.

Proof. That $\operatorname{dim} X_{0}^{+}<\infty$ follows from condition (ii) since $A(0)$ has compact resolvent.

For the second part, we begin with the remark that, by (i), $D_{A(0)} u=0$ on $\mathbf{R}_{-}$ and that $u\left(t_{0}\right) \in X$ is well defined since $\mathcal{W}^{p} \subset W^{1, p}(\mathbf{R}, X) \subset C^{0}(\mathbf{R}, X)$. Denote by $P^{+} \in \mathcal{L}(X)$ the spectral projection onto $X_{0}^{+}$, so that $X=X_{0}^{+} \oplus X_{0}^{-}$, where $X_{0}^{-}:=P^{-}(X)$ and $P^{-}:=I-P^{+}$. Set $u^{-}:=P^{-} u$. Notice that $X_{0}^{+} \subset W$ and that $P^{-}(W) \subset W$, so that $P^{-}$and $A(0)$ commute and hence $u^{-}:=P^{-} u \in \mathcal{W}^{p}$ and $u^{-}$ satisfies

$$
\frac{d u^{-}}{d t}-A(0) u^{-}=0 \text { on } \mathbf{R}_{-} .
$$

To prove that $u\left(t_{0}\right) \in X_{0}^{+}$for every $t_{0}<0$, it suffices to show that $\left(u^{-}\right)_{\mid\left(-\infty, t_{0}\right]}$ can be extended to $\tilde{u}^{-} \in \mathcal{W}^{p}$ such that $D_{A(0)} \tilde{u}^{-}=0$. If so, $\tilde{u}^{-}=0$ by Theorem 2.2 for $A(0)$, so that $u^{-}\left(t_{0}\right)=\tilde{u}^{-}\left(t_{0}\right)=0$. That $u(0) \in X_{0}^{+}$then follows by continuity since $X_{0}^{+}$is closed in $X$. To see that $A(0)$ does satisfy the hypotheses of Theorem 2.2 , recall that the norm of $W=D(A(0))$ is equivalent to the graph norm of $A(0)$ and note that $\sigma(A(0)) \cap i \mathbf{R}=\emptyset$ by (H5) since $A(0)=A_{-}$by condition (i) of the lemma. Of course, (H4) with $t=0$ is just (1.3).

We thus pass to the proof that $\left(u^{-}\right)_{\mid\left(-\infty, t_{0}\right]}$ has the desired extension property when $t_{0}<0$. Consider the autonomous initial value problem

$$
\left\{\begin{array}{l}
\frac{d v}{d t}=A(0) v \text { in }\left(t_{0}, \infty\right), \\
v\left(t_{0}\right)=u^{-}\left(t_{0}\right)
\end{array}\right.
$$

with the solution

$$
v(t)=S_{0}\left(t-t_{0}\right) u^{-}\left(t_{0}\right), \quad \forall t \geq t_{0},
$$

where $S_{0}$ is the semigroup generated by $A(0)$. 
Our first task will be to show that not only $u\left(t_{0}\right) \in X$ but in fact $u\left(t_{0}\right) \in W$. Since $P^{-}(W) \subset W$, this shows that $u^{-}\left(t_{0}\right) \in W$, which endows the solution $v$ in (8.12) with better properties. Set $f:=D_{A} u \in \mathcal{X}^{p}$ and choose $\varphi \in \mathcal{D}(\mathbf{R})$ with $\operatorname{Supp} \varphi \subset \mathbf{R}_{-}$and $\varphi\left(t_{0}\right)=1$. By multiplying $D_{A} u=f$ by $\varphi$, we obtain $D_{A}(\varphi u)=\varphi f+u \frac{d \varphi}{d t}=u \frac{d \varphi}{d t}$ since $f=0$ a.e. on $\operatorname{Supp} \varphi \subset \mathbf{R}_{-}$. Also, by condition (i) of the lemma and $\operatorname{Supp}(\varphi u) \subset \mathbf{R}_{-}$, we have $D_{A}(\varphi u)=D_{A(0)}(\varphi u)$. Thus,

$$
D_{A(0)}(\varphi u)=u \frac{d \varphi}{d t} \in \mathcal{W}^{p} \subset W^{1, p}(\mathbf{R}, X)
$$

and by Remark 2.1 for $A(0)$, it follows that $\varphi u \in W^{1, p}(\mathbf{R}, W) \subset C^{0}(\mathbf{R}, W)$. In particular, $\varphi\left(t_{0}\right) u\left(t_{0}\right)=u\left(t_{0}\right) \in W$, as claimed.

Note that, in (8.12), $S_{0}$ may be viewed as a holomorphic semigroup on the closed invariant subspace $X_{0}^{-}$. By condition (H5) for $A_{-}=A(0)$ and the compactness of the resolvent of $A(0)$, it follows that $S_{0}$ is a bounded holomorphic semigroup on $X_{0}^{-}$generated by $A(0)_{\mid X_{0}^{-}}$. Thus, sup $\operatorname{Re} \sigma\left(A(0)_{\mid X_{0}^{-}}\right)<0$, so that $\left\|S_{0}(t)\right\|_{\mathcal{L}\left(X_{0}^{-}\right)}$has exponential decay on $[0, \infty)$ (Pazy [30, Theorem 4.3, p. 118]).

By (8.12) and the exponential decay of $\left\|S_{0}(t)\right\|_{\mathcal{L}\left(X_{0}^{-}\right)}$, it follows at once that $v \in$ $L^{p}\left(\left(t_{0}, \infty\right), X_{0}^{-}\right) \subset L^{p}\left(\left(t_{0}, \infty\right), X\right)$. In addition, since $u^{-}\left(t_{0}\right) \in W=D(A(0))$, we have $v(t) \in D(A(0))$ for $t \geq t_{0}$ and $A(0) v(t)=A(0) S_{0}(t) u^{-}\left(t_{0}\right)=S_{0}(t) A(0) u^{-}\left(t_{0}\right)$. Thus, $A(0) v \in L^{p}\left(\left(t_{0}, \infty\right), X\right)$, once again by exponential decay, and then $\frac{d v}{d t} \in$ $L^{p}\left(\left(t_{0}, \infty\right), X\right)$ by $(8.11)$. Since the norm of $W$ is equivalent to the graph norm of $A(0)$, the relations $v \in L^{p}\left(\left(t_{0}, \infty\right), X\right)$ and $A(0) v \in L^{p}\left(\left(t_{0}, \infty\right), X\right)$ amount to $v \in L^{p}\left(\left(t_{0}, \infty\right), W\right)$. Altogether, we have obtained

$$
v \in W^{1, p}\left(\left(t_{0}, \infty\right), X\right) \cap L^{p}\left(\left(t_{0}, \infty\right), W\right) .
$$

From $(8.10),(8.11)$ and $(8.13)$, it is clear that the function $\tilde{u}^{-} \in C^{0}(\mathbf{R}, X)$ defined by $\tilde{u}^{-}=u$ on $\left(-\infty, t_{0}\right]$ and $\tilde{u}^{-}=v$ on $\left(t_{0}, \infty\right)$ satisfies $\tilde{u}^{-} \in \mathcal{W}^{p}$ and $D_{A(0)} \tilde{u}^{-}=0$ on $\mathbf{R}$, as desired.

The "in particular" part is obvious since $X_{0}^{+}=\{0\}$ when $A(0)$ generates a bounded holomorphic semigroup. This completes the proof.

LEMMA 8.3. Let $X$ be a Banach space with UMD and let the family $(A(t))_{t \in \mathbf{R}}$ of unbounded operators on $X$ satisfy not only the assumptions (H1) to (H5) but also

(i) $A(t)=A(0)$ for every $t \leq 0$.

(ii) $A(0)\left(=A_{-}\right)$generates a bounded holomorphic semigroup on $X$.

(iii) $A(t)$ generates a holomorphic semigroup on $X$ for every $t \geq 0$.

Then, given $p \in(1, \infty)$, the operator $D_{A}:=\frac{d}{d t}-A$ is Fredholm and one-to-one from $\mathcal{W}^{p}$ to $\mathcal{X}^{p}$ (so that index $\left.D_{A}=-\nu \leq 0\right)$. In addition, if $g_{1}^{*}, \ldots, g_{\nu}^{*} \in \mathcal{X}_{*}^{p^{\prime}}$ is a basis of ker $D_{-A^{*}}$ when $D_{-A^{*}}$ is viewed as a linear operator from $\mathcal{X}_{*}^{p^{\prime}}$ to $\mathcal{W}_{*}^{p^{\prime}}$ (see (4.1) and Theorem 4.2), then the restrictions of $g_{1}^{*}, \ldots, g_{\nu}^{*}$ to $\mathbf{R}_{+}$remain linearly independent in $L^{p^{\prime}}\left(\mathbf{R}_{+}, X^{*}\right)$.

Proof. That $D_{A}$ is Fredholm follows from Theorem 2.3. To prove that $D_{A}$ is one-to-one, let $u \in \mathcal{W}^{p}$ be such that $D_{A} u=0$. By Lemma $8.2, u=0$ on $\overline{\mathbf{R}}_{-}$, so that $u \in W^{1, p}\left(\mathbf{R}_{+}, X\right) \cap L^{p}\left(\mathbf{R}_{+}, W\right)$ solves the initial value problem

$$
\left\{\begin{array}{l}
\frac{d u}{d t}=A u \text { in } \mathbf{R}_{+}, \\
u(0)=0,
\end{array}\right.
$$


whose only solution is $u=0$ by Lemma 8.1 (ii). This shows that $u=0$ on $\mathbf{R}$.

Next, let $g^{*}=\sum_{i=1}^{\nu} \mu_{i} g_{i}^{*}$ be a linear combination such that $g^{*}=0$ in $L^{p^{\prime}}\left(\mathbf{R}_{+}, X^{*}\right)$ and hence a.e. on $\mathbf{R}_{+}$. Since $g^{*} \in \mathcal{X}_{*}^{p^{\prime}} \subset C^{0}\left(\mathbf{R}, W^{*}\right)$, it follows that $g^{*}(t)=0$ for all $t \geq 0$. In particular, $g^{*}(0)=0$. Since also $g^{*} \in \operatorname{ker} D_{-A^{*}}$ means that $\frac{d g^{*}}{d t}=-A^{*} g^{*}$, it follows from Remark 8.1 for $-A^{*}$ that $g^{*}=0$ in $\mathbf{R}_{-}$(and hence $g^{*}=0$, so that $\left.\mu_{1}=\cdots=\mu_{\nu}=0\right)$, provided that $A^{*}(t)$ generates a holomorphic semigroup on $W^{*}$ for all $t \leq 0$.

Since $A(t)=A(0)$ for all $t \leq 0$, this amounts to showing that $A^{*}(0)$ generates a holomorphic semigroup on $W^{*}$. Because this statement involve $W^{*}$ and not $X^{*}$, it is not the classical one and some justification is needed, which is as follows. Since $A(0)$ generates a bounded holomorphic semigroup on $X$ and $0 \notin \sigma(A(0))$, there is a constant $M>0$ such that $\|R(\lambda, A(0))\|_{\mathcal{L}(X)} \leq \frac{M}{|\lambda|+1}$ for $\lambda \in \mathbf{C}$ in some large enough sector $\Sigma$ containing the positive real axis. Since $W$ is equipped with the graph norm of $A(0)$, this implies $\|R(\lambda, A(0))\|_{\mathcal{L}(X, W)} \leq M$ after possibly changing $M$ and then $\left\|R\left(\lambda, A^{*}(0)\right)\right\|_{\mathcal{L}\left(W^{*}, X^{*}\right)}=\left\|R(\lambda, A(0))^{*}\right\|_{\mathcal{L}\left(W^{*}, X^{*}\right)} \leq M$. (Here, we used the fact that the embedding $X^{*} \hookrightarrow W^{*}$ is the adjoint of the embedding $W \hookrightarrow X$.) In turn, since the norm of $X^{*}$ is equivalent to the graph norm of $A^{*}(0)$ (this was seen in the proof of Theorem 4.2), this yields, after changing $M$ once again if necessary, that $\left\|R\left(\lambda, A^{*}(0)\right)\right\|_{\mathcal{L}\left(W^{*}\right)} \leq \frac{M}{|\lambda|+1}$ for $\lambda \in \Sigma$, which proves the claim.

THEOREM 8.4. Let $X$ be a Banach space with UMD and let the family $(A(t))_{t \in \mathbf{R}}$ of unbounded operators on $X$ satisfy not only the assumptions (H1) to (H5) but also (i) $A(t)=A(0)$ for every $t \leq 0$ (so that $A_{-}=A(0)$ ).

(ii) $A(0)\left(=A_{-}\right)$generates a bounded holomorphic semigroup on $X$.

(iii) $A(t)$ generates a holomorphic semigroup on $X$ for every $t \geq 0$.

For $p \in(1, \infty)$, let $-\nu \leq 0$ denote the index ${ }^{9}$ of $D_{A}: \mathcal{W}^{p} \rightarrow \mathcal{X}^{p}$ (see Lemma 8.3). Then, $\nu$ equals the dimension of the sum of the generalized eigenspaces of $A_{+}$corresponding to eigenvalues with positive real part. Furthermore, there are $\nu$ linearly independent elements $g_{1}^{*}, \ldots, g_{\nu}^{*} \in L^{p^{\prime}}\left(\mathbf{R}_{+}, X^{*}\right)$ with the following property: Given $f \in L^{p}\left(\mathbf{R}_{+}, X\right)$, the initial value problem

$$
\left\{\begin{array}{l}
\frac{d u}{d t}=A u+f \text { in } \mathbf{R}_{+}, \\
u(0)=0,
\end{array}\right.
$$

has a solution $u \in W_{0}^{1, p}\left(\mathbf{R}_{+}, X\right) \cap L^{p}\left(\mathbf{R}_{+}, W\right)$ if and only if $\int_{0}^{\infty}\left\langle f, g_{i}^{*}\right\rangle_{X, X^{*}}=0$ for $1 \leq i \leq \nu$. If so, the solution $u$ is unique and there is a constant $C>0$ independent of $f$ such that

$$
\|u\|_{W^{1, p}\left(\mathbf{R}_{+}, X\right)}+\|u\|_{L^{p}\left(\mathbf{R}_{+}, W\right)} \leq C\|f\|_{L^{p}\left(\mathbf{R}_{+}, X\right)} .
$$

PRoOF. The characterization of $\nu$ follows from Theorem 6.7 since, here, $\nu_{-}=0$ by (i) and (ii). If $u$ exists, then the extension $\tilde{u}$ of $u$ by 0 in $\mathbf{R}_{-}$is in $\mathcal{W}^{p}$ and solves $D_{A} \tilde{u}=\tilde{f}$ where $\tilde{f} \in \mathcal{X}^{p}$ is the extension of $f$ by 0 in $\mathbf{R}_{-}$. From Theorem 4.2 , this is possible if and only if $\int_{\mathbf{R}}\left\langle\tilde{f}, g_{i}^{*}\right\rangle_{X, X^{*}}=0$ for $1 \leq i \leq \nu$ where $g_{1}^{*}, \ldots, g_{\nu}^{*} \in \mathcal{X}^{p^{\prime}}$ is a basis of $\operatorname{ker} D_{-A^{*}}$. Since $\tilde{f}=0$ on $\mathbf{R}_{-}$, this condition reduces to $\int_{0}^{\infty}\left\langle f, g_{i}^{*}\right\rangle_{X, X^{*}}=0$. By Lemma $8.3, \nu=$ index $D_{A}, g_{1}^{*}, \ldots, g_{\nu}^{*}$ remain linearly independent in $L^{p^{\prime}}\left(\mathbf{R}_{+}, X^{*}\right)$ and the solution $\tilde{u}$ (hence also $u$ ) is unique.

${ }^{9}$ Independent of $p$ by Theorem 5.1. 
Conversely, by reversing the above steps, we find that if $\int_{0}^{\infty}\left\langle f, g_{i}^{*}\right\rangle_{X, X^{*}}=0$ for $1 \leq i \leq \nu$, then $\int_{\mathbf{R}}\left\langle\tilde{f}, g_{i}^{*}\right\rangle_{X, X^{*}}=0$ and hence there is a unique $\tilde{u} \in \mathcal{W}^{p}$ such that $D_{A} \tilde{u}=\tilde{f}$. But then $\tilde{u}=0$ in $\overline{\mathbf{R}}_{-}$by Lemma 8.2 , so that $u=\tilde{u}_{\mid \mathbf{R}_{+}}$ satisfies (8.14). All this shows that the operator $\frac{d}{d t}-A$ is an isomorphism of $W_{0}^{1, p}\left(\mathbf{R}_{+}, X\right) \cap L^{p}\left(\mathbf{R}_{+}, W\right)$ onto the closed subspace of $L^{p}\left(\mathbf{R}_{+}, X\right)$ of those $f$ such that $\int_{0}^{\infty}\left\langle f, g_{i}^{*}\right\rangle_{X, X^{*}}=0$ for $1 \leq i \leq \nu$. The existence of a constant $C>0$ such that (8.15) holds is a mere translation of this property.

In practice, the operators $A(t)$ are only given for $t \geq 0$, but it suffices to define $A(t)=A(0)$ for $t<0$ to be in a position to use Theorem 8.4. Clearly, it answer the $L^{p}$ maximal regularity question in the nonautonomous case when $A(0)$ generates a bounded holomorphic semigroup (otherwise, see Corollary 8.5 below). The relevance of the functions $g_{1}^{*}, \ldots, g_{\nu}^{*}$ (depending upon the operator $D_{-A^{*}}$ on the whole line) is a new feature, even when $p=2$ and $X$ and $W$ are Hilbert spaces.

As a corollary, we now obtain a generalization of Theorem 8.4 when condition (ii) and (H5) for $A_{-}=A(0)$ are dropped. To our best knowledge, this was previously known only when $A(t)=A$ is constant and $n u=0$.

Corollary 8.5. Let $X$ be a Banach space with UMD and let the family $(A(t))_{t \in \mathbf{R}}$ of unbounded operators on $X$ satisfying the assumptions (H1) to (H4) and also

(i) $A(t)=A(0)$ for every $t \leq 0$ (so that $A_{-}=A(0)$ ).

(ii) $\sigma\left(A_{+}\right) \cap i \mathbf{R}=\emptyset$.

(iii) A(t) generates a holomorphic semigroup on $X$ for every $t \geq 0$.

Let $\nu \geq 0$ denote the dimension of the sum of the generalized eigenspaces of $A_{+}$ corresponding to eigenvalues with positive real part. Then, $\nu<\infty$ and for every $p \in(1, \infty)$, the operator

$$
\frac{d}{d t}-A: W_{0}^{1, p}\left(\mathbf{R}_{+}, X\right) \cap L^{p}\left(\mathbf{R}_{+}, W\right) \rightarrow L^{p}\left(\mathbf{R}_{+}, X\right),
$$

is one to one and Fredholm of index $-\nu$. In particular, it is an isomorphism if $\nu=0$.

Proof. Given $\delta>0$, let $\varphi: \overline{\mathbf{R}}_{+} \rightarrow\left[\frac{1}{2}, 1\right]$ be a smooth function such that $\varphi(0)=1, \dot{\varphi}(0)=-\delta$ and $\varphi(t)=1$ for $t \geq 1$. Then, the multiplication by $\varphi$ is an isomorphism of $W_{0}^{1, p}\left(\mathbf{R}_{+}, X\right) \cap L^{p}\left(\mathbf{R}_{+}, W\right)$ and of $L^{p}\left(\mathbf{R}_{+}, X\right)$, with inverse the multiplication by $\frac{1}{\varphi}$. It follows that the Fredholm properties (and index) of the operator (8.16) are unchanged when $A$ is replaced by $A_{\varphi}$, where $A_{\varphi}(t):=$ $A(t)-\frac{\dot{\varphi}(t)}{\varphi(t)} I$. Now, $A_{\varphi}(t)$ has exactly the same properties as $A(t)$ and even coincides with it for $t \geq 1$, so that $A_{+}$is unchanged, except that $A_{\varphi}(0)=A(0)-\delta I$. (That $A_{\varphi}$ satisfies (H4) follows from (H4) for $A$ and Theorem 5.3 since the embedding of $W$ into $X$ is compact.) Clearly, if $\delta>0$ is large enough, then $\sigma\left(A_{\varphi}(0)\right) \cap i \mathbf{R}=\emptyset$ and $A_{\varphi}(0)$ generates a bounded holomorphic semigroup. The conclusion follows from Theorem 8.4 for $A_{\varphi}$.

Naturally, Corollary 8.5 implies that (8.15) continues to hold for every righthand side $f$ for which $\frac{d u}{d t}=A u+f$ is solvable.

It is obvious how to derive an existence and uniqueness result for the problem (8.2) from Corollary 8.5 when the initial condition $u(0)=0$ is replaced by $u(0)=$ $u_{0} \in W$, by using a lifting of $u_{0}$ in $W^{1, p}\left(\mathbf{R}_{+}, X\right) \cap L^{p}\left(\mathbf{R}_{+}, W\right)$. Actually, it is clear 
that the "right" space for $u_{0}$ is not $W$ but, rather, the space of traces $\{u(0): u \in$ $\left.W^{1, p}\left(\mathbf{R}_{+}, X\right) \cap L^{p}\left(\mathbf{R}_{+}, W\right)\right\}$, intermediate between $W$ and $X$. We do not know if this space coincides with more classical interpolation spaces between $W$ and $X$, but it contains the real interpolation space $(X, W)_{1-\frac{1}{p}, p}$; see [16, Lemma 2.1].

It is also a simple exercise to obtain the $L^{p}$ maximal regularity over finite intervals from Corollary 8.5). In this problem, a family $A(t)$ is given for $t \in[0, T]$ with $T \in(0, \infty)$ and then extended by setting $A(t)=A(0)$ for $t<0$ and $A(t)=$ $A(T)$ for $t>T$, so that $A_{-}=A(0)$ and $A_{+}=A(T)$. This extended family is assumed to satisfy the assumptions of Corollary 8.5. Since attention is confined to solutions on $(0, T)$ and right-hand sides in $L^{p}((0, T), X)$ (i.e., in $L^{p}\left(\mathbf{R}_{+}, X\right)$ that vanish a.e. in $(T, \infty))$ and since the multiplication by $e^{\mu t}$ is an isomorphism of all three spaces $L^{p}((0, T), X), W_{0}^{1, p}((0, T), X)$ and $L^{p}((0, T), W), A(t)$ may be changed into $A(t)-\mu I$ for any $\mu>0$ upon setting $u=e^{\mu t} v$. Thus, the problem can always be reduced to the case when $A(T)=A_{+}$generates a bounded holomorphic semigroups, so that $\nu=0$ and the isomorphism property of Corollary 8.5 can be used (no compatibility condition arises).

REMARK 8.2. According to [33], the Fredholm property of (8.16) has an immediate impact on the asymptotic behavior of the solutions $u$ of (8.2) depending upon the asymptotic behavior of $f \in L^{p}\left(\mathbf{R}_{+}, X\right)$. See also Schnaubelt [35] for other results of this type, by a different approach.

\section{References}

[1] Abbondandolo, A. and Majer, P., Ordinary differential operators in Hilbert spaces and Fredholm pairs, Math. Zeit. 243 (2003) 525-562.

[2] Acquistapace, P. and Terreni, B., A unified approach to abstract linear nonautonomous parabolic equations, Rend. Sem. Mat. Univ. Padova 78 (1987) 47-107.

[3] Abraham, R., Marsden, J. E. and Ratiu, T., Manifolds, tensor analysis and applications, 2nd edition, Applied Mathematical Sciences, vol. 75, Springer-Verlag, New York (1988).

[4] Abraham, R. and Robbin, J., Transversal mappings and flows, Addison-Wesley, Reading Mass. 1967

[5] Aldous, D. J., Unconditional bases and martingales in $L_{p}(F)$, Math. Proc. Cambridge Philos. Soc. 85 (1979) 117-123.

[6] Amann, H., Hieber, M. and Simonett, G., Bounded $H_{\infty}$-calculus for elliptic operators, Differ. Integral Eq. 7 (1994), 613-653.

[7] Arendt, W. and ter Elst, A. F. M., Gaussian estimates for second order elliptic operators with boundary conditions, J. Operator Theory 38 (1997) 87-130.

[8] Atiyah, M. F., Patodi, V. K. and Singer, I. M., Spectral asymmetry and Riemannian geometry I, II, III, Math. Proc. Cambridge Philos. Soc. 77 (1975) 43-69, 78 (1975) 405-432, 79 (1976) 71-99.

[9] Bourgain, J., Some remarks on Banach spaces in which martingale differences are unconditional, Arkiv. Math. 21 (1983) 163-168.

[10] Bourgain, J., Vector-valued singular integrals and the H-BMO duality, Probability Theory and Harmonic Analysis, Dekker, New York (1985) 1-19.

[11] Burkholder, D. L., Martingale transforms, Ann. Math. Statist. 37 (1966) 1494-1504.

[12] Burkholder, D. L., A geometrical characterization of Banach spaces in which martingale difference sequences are unconditional, Ann. Probab. 9 (1981) 997-1011.

[13] Burkholder, D. L., A geometric condition that implies the existence of certain singular integrals of Banach-space-valued functions, Conference on harmonic analysis in honor of Antoni Zygmund, Vol. I, II, Wadsworth Math. Ser., Wadsworth, Belmont (1983).

[14] Deimling, K., Nonlinear Functional Analysis, Springer-Verlag, Berlin 1985.

[15] Di Giorgio, D. and Lunardi, A., On Fredholm properties of $L u=u^{\prime}-A(t) u$ for paths of sectorial operators, Proc. Royal Soc. Edinburgh (to appear). 
[16] Di Giorgio, D., Lunardi, A. and Schnaubelt, R., Fredholm properties of abstract parabolic operators in $L^{p}$ spaces on the real line (preprint).

[17] Dore, G. and Venni, A., On the closedness of the sum of two closed operators, Math. Z. 196 (1987) 189-201.

[18] Duong, X. T. and McIntosh, A., Functional calculi of second-order elliptic partial differential operators with bounded measurable coefficients, J. Geom. Anal. 6 (1996), 181-205.

[19] Duong, X. T. and Robinson, D. W., Semigroup kernels, Poisson bounds and holomorphic functional calculus, J. Funct. Anal. 142 (1996) 89-129.

[20] Edwards, R. E., Functional Analysis, Theory and Applications, Holt, Rinehart and Winston, New York, 1965.

[21] Giga, M., Giga, Y. and Sohr, H., $L_{p}$ estimate for abstract linear parabolic equations, Proc. Japan Acad. Ser. A 67 (1991) 197-202.

[22] Hess, P., Zur Störungstheorie linearer Operatoren: Relative Beschränktheit und relative Kompaktheit von Operatoren in Banachräumen, Comment. Math. Helv. 44 (1969) 245-248.

[23] Hieber, M. and Monniaux, S., Heat-kernels and maximal $L_{p}-L_{q}$ estimates: the nonautonomous case, J. Fourier Anal. Appl. 6 (2000) 467-481.

[24] Kalton, N. J. and Weis, L., The $H^{\infty}$-calculus and sums of closed operators, Math. Ann. 321 (2001) 319-345.

[25] Kato, T., Perturbation Theory for Linear Operators, Springer-Verlag, Berlin, 1980.

[26] Latushin, Y. and Tomilov, Y., Fredholm differential operators with unbounded coefficients (preprint).

[27] Lindenstrauss, J. and Tzafriri, L., Classical Banach Spaces I and II, Springer-Verlag, Berlin, 1996.

[28] Maurey, B., Système de Haar, Séminaire Maurey-Schwartz 1974-1975, Ecole Polytechnique, Paris (1975).

[29] Monniaux, S. and Prüss, J., A theorem of the Dore-Venni type for noncommuting operators, Trans. Amer. Math. Soc. 349 (1997) 4787-4814.ger-Verlag, New York, 1983.

[30] Pazy, A., Semigroups of Linear Operators and Applications to Partial Differential Equations, Applied Mathematical Sciences, Vol. 44, Springer-Verlag, New York, 1983.

[31] Prüss, J. and Schnaubelt, R., Solvability and maximal regularity of parabolic evolution equations with coefficients continuous in time, J. Math. Anal. Appl. 256 (2001) 405-430.

[32] Rabier, P. J., An isomorphism theorem for linear evolution problems on the line, J. Dyn. Diff. Eq. 15 (2003) 779-806.

[33] Rabier, P. J., Fredholm operators, semigroups and the asymptotic and boundary behavior of solutions of PDEs, J. Differ. Eq. 193 (2003) 460-480.

[34] Robbin, J. and Salamon, D., The spectral flow and the Maslov index, Bull. London Math. Soc. 27 (1995) 1-33.

[35] Schnaubelt, R. Asymptotically autonomous parabolic evolution equations, J. Evol. Eq. 1 (2001), 19-37.

[36] de Simon, L., Un'applicazione della teoria degli integrali singolari allo studio delle equazioni differenziali lineari astratte del primo ordine, Rend. Sem. Mat. Univ. Padova 34 (1964) 205-223.

[37] Simon, J., Compact sets in the space $L^{p}(0, T ; B)$, Ann. Mat. Pura Appl. 146 (1987) 65-96.

[38] Weis, L., Operator-valued Fourier multiplier theorems and maximal $L_{p}$ regularity, Math. Ann. 319 (2001) 735-758.

[39] Yamamoto, Y., Solutions in $L_{p}$ of abstract parabolic equations in Hilbert spaces, J. Math. Kyoto Univ. 33 (1993) 299-314.

[40] Zimmermann, F., On vector-valued Fourier multiplier theorems, Studia Math. 93 (1989) 201-222.

Department of Mathematics, University of Pittsburgh, Pittsburgh, PA 15260

E-mail address: rabier@imap.pitt.edu 